Supporting Information

\title{
Solvent-free, mechanochemically scalable synthesis of 2,3-dihydroquinazolin-4(1H)-one using Brønsted acid catalyst
}

\author{
Gauravi Yashwantrao, ${ }^{\dagger}$ Valmik P. Jejurkar, ${ }^{\dagger}$ Rajpratap Kshatriya and Satyajit Saha* \\ Department of Dyestuff Technology, Institute of Chemical Technology, Matunga, Mumbai, \\ Maharashtra-400019, India.
}

\section{Contents}

General Aspects. ... S2

General Method For The Preparation Of The 2,3-dihydroquinazolin-4(1H)-one (3a-3o, and 8S2 11)

General procedure for the preparation of bisquinazolinone (13a-b)

Melting points of the synthesized 2,3-dihydroquinazolin-4(1H)-one S3-S4

3a-3o, 8-11 and 13 and comparison with the literature reported data.

Characterization data of the 2,3-dihydroquinazolin-4(1H)-one derivatives.

S5-S8

Reproducibility of the reaction between anthranilamide $\mathbf{1 a}$ and benzaldehyde S8

2a......

Solubility profile of anthranilamide and benzaldehyde in molten $p$-TSA. S8

Calculation Of The Green Chemistry Metrics.

Process Intensification: Mechanochemical scalable synthesis of 2-(4-nitrophenyl)-2,3S10-S12 dihydroquinazolin-4(1H)-one (3h) by ball milling. ...

${ }^{1} \mathrm{H}$ and ${ }^{13} \mathrm{C}$ NMR Spectral reproductions of representative 2,3-dihydroquinazolin-4(1H)-one (3a, 3c, 3d, 3h, 8b, 9b, 10a, 10b, 10c, 11b and 13b

References.

\section{Number of pages: $\mathbf{2 4}$ \\ Number of Figures: 16 \\ Number of schemes: 4 \\ Number of Tables: $\mathbf{5}$}




\section{General Aspects}

All chemicals were received from Sigma Aldrich, Spectrochem, S.D Fine chemicals, Avra chemicals and used as such without further purification. The reactions were monitored by TLC carried out on Merck silica gel (60F254) by using UV light and phosphomolybdic acid as visualizing and developing agents. Proton nuclear magnetic resonance spectra $\left({ }^{1} \mathrm{H} \mathrm{NMR}\right)$ were obtained at $400 \mathrm{MHz}$ on Agilent spectrometer. ${ }^{13} \mathrm{C}$ NMR spectra were obtained at $100 \mathrm{MHz}$ on Agilent spectrometer. Spectra were recorded in $\mathrm{CDCl}_{3}$ and DMSO- $d_{6}$ solutions. Chemical shifts are reported in ppm, referenced to tetramethylsilane (TMS) as the external reference. Hydrogen coupling patterns are described as singlet $(\mathrm{s})$, doublet (d), triplet ( $\mathrm{t})$ and multiplet (m). Coupling constants (J) are reported in Hertz. Melting points of all the compounds were recorded by Analab ThermoCal melting point apparatus in the open capillary tube. All grinding reactions are carried out in mortar and pestle at room temperature. Ball milling was performed in tumbler ball milling instrument purchased from S.F. Engineering works having frequency $40 \mathrm{rpm}$, voltage $230 \mathrm{~V}$, motor power $18.5 \mathrm{~kW}$, and current $24 \mathrm{~A}$. Internal diameter of milling vessel was $5 \mathrm{~cm}$ with $200 \mathrm{ml}$ capacity.

\section{General Method For The Preparation Of The 2,3-dihydroquinazolin-4(1H)-one (3a-3o, and 8-11)}

Scheme S1. Reaction of anthranilamides (1a-c) with aldehydes (2a-o) or ketones (4-7) under grinding in mortar pestle.

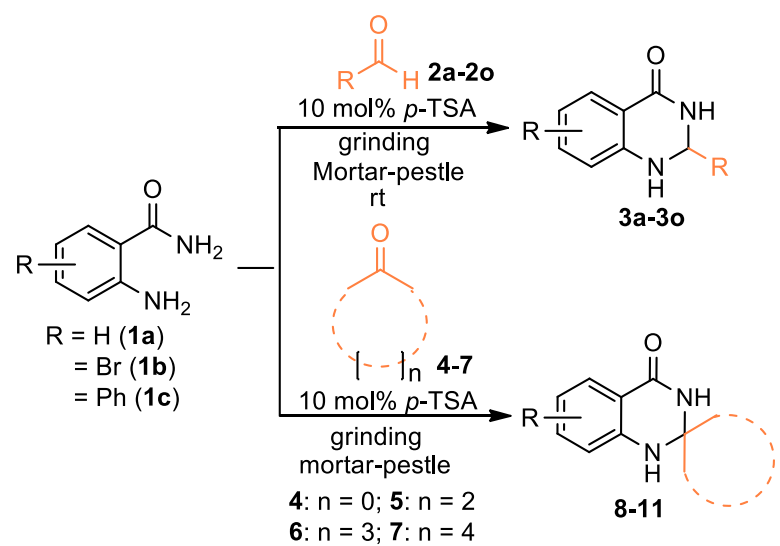

Procedure: To a mixture of anthranilamide $1(0.100 \mathrm{~g}, 0.73 \mathrm{mmol}, 1$ equiv. $)$, and $p$-nitrobenzaldehyde $2(0.122 \mathrm{~g}, 0.81 \mathrm{mmol}, 1.1$ equiv.) was added $p$-TSA catalyst $(10 \mathrm{~mol} \%)$ and ground in a mortar-pestle at room temperature. Upon completion of the reaction, as monitored by TLC, water was added to the reaction mixture to remove the $p$-TSA, and the product was filtered off. The residual solid was washed with water and then with $2 \%$ EtOAc-hexane mixture to remove excess aldehyde and to isolate $0.183 \mathrm{~g}$ (93\%) of the desired product.

Similar procedure was followed for the synthesis of the compounds 3a-3o and 8-11. 


\section{General procedure for the preparation of bisquinazolinone (13a-b)}

Scheme S2. Reaction of anthranilamides (1a-b) with terephthaldehyde (12) under grinding in mortar pestle.

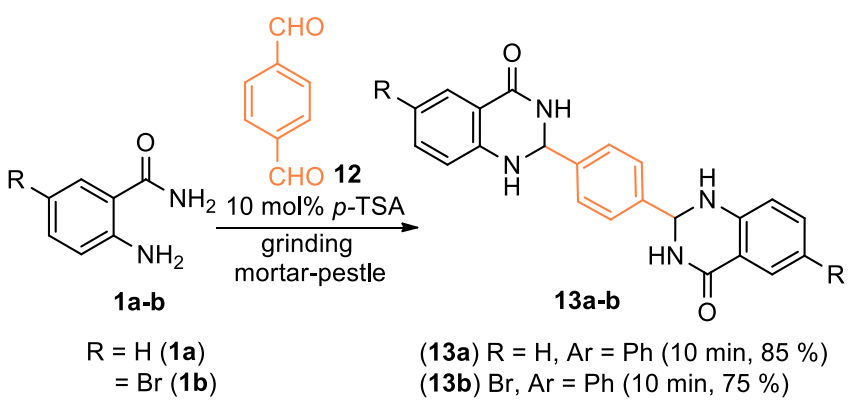

Procedure: To a mixture of terephthaldehyde $12(0.100 \mathrm{~g}, 0.74 \mathrm{mmol}, 1$ equiv.) and anthranilamide 1 $(0.223 \mathrm{~g}, 1.64 \mathrm{mmol}, 2.2$ equiv.) was $10 \mathrm{~mol} \%$ of $p$-TSA catalyst and ground in a mortar-pestle. The progress of the reaction was monitored by TLC. Upon completion of the reaction, water was added to remove the $p$-TSA, and the product was filtered off. The residual white solid was washed with water and then purified by silica gel column chromatography using DCM:MeOH as eluent to get hold of 0.22 $\mathrm{g}(80 \%)$ of the desired product.

Melting points of the synthesized 2,3-dihydroquinazolin-4(1H)-one 3a-3o, 8-11 and 13 and comparison with the literature reported data

Table S1. Melting point of the synthesized 2,3-dihydroquinazolin-4(1H)-one 3a-3o, 8-11 and 13

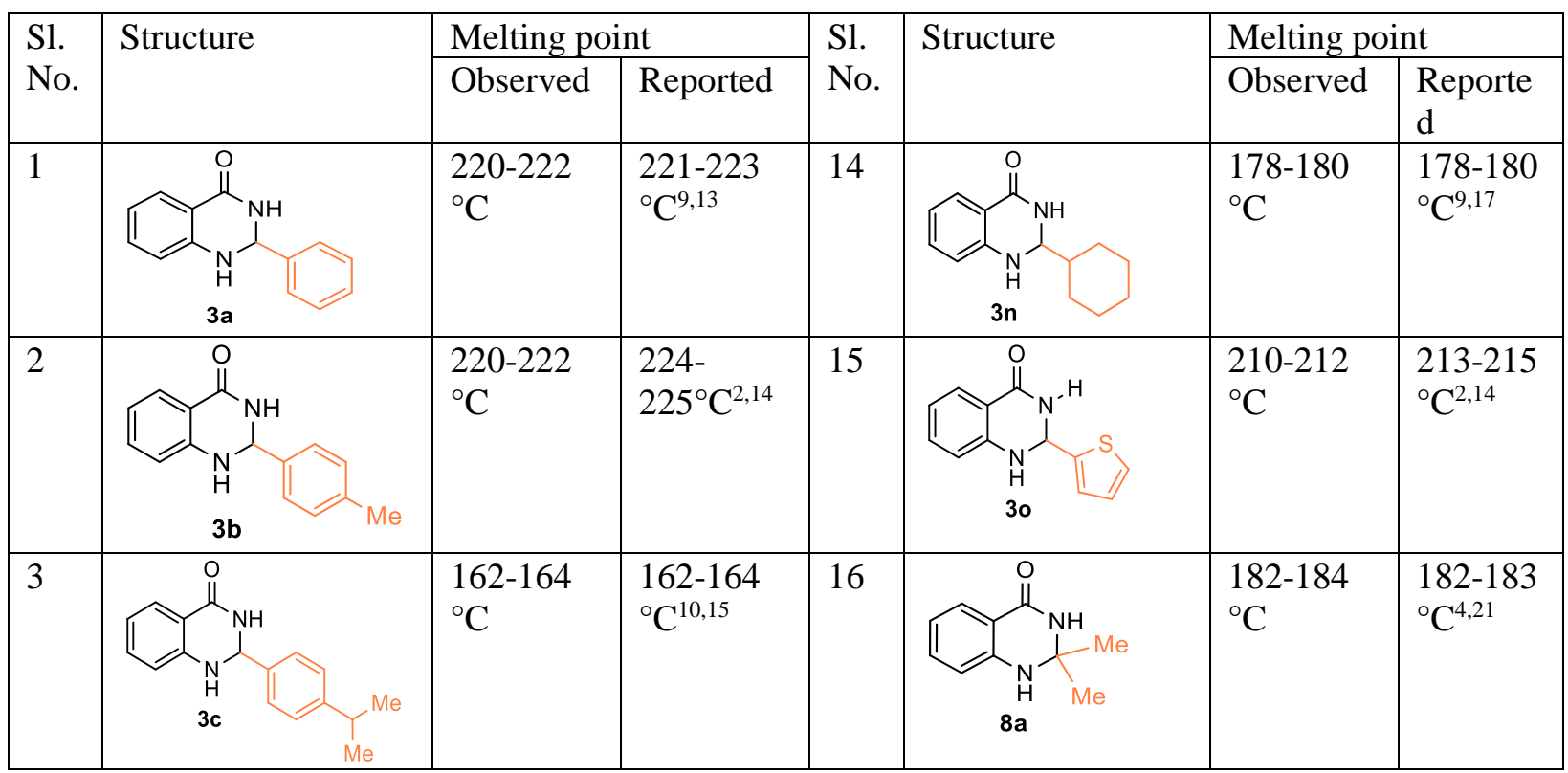




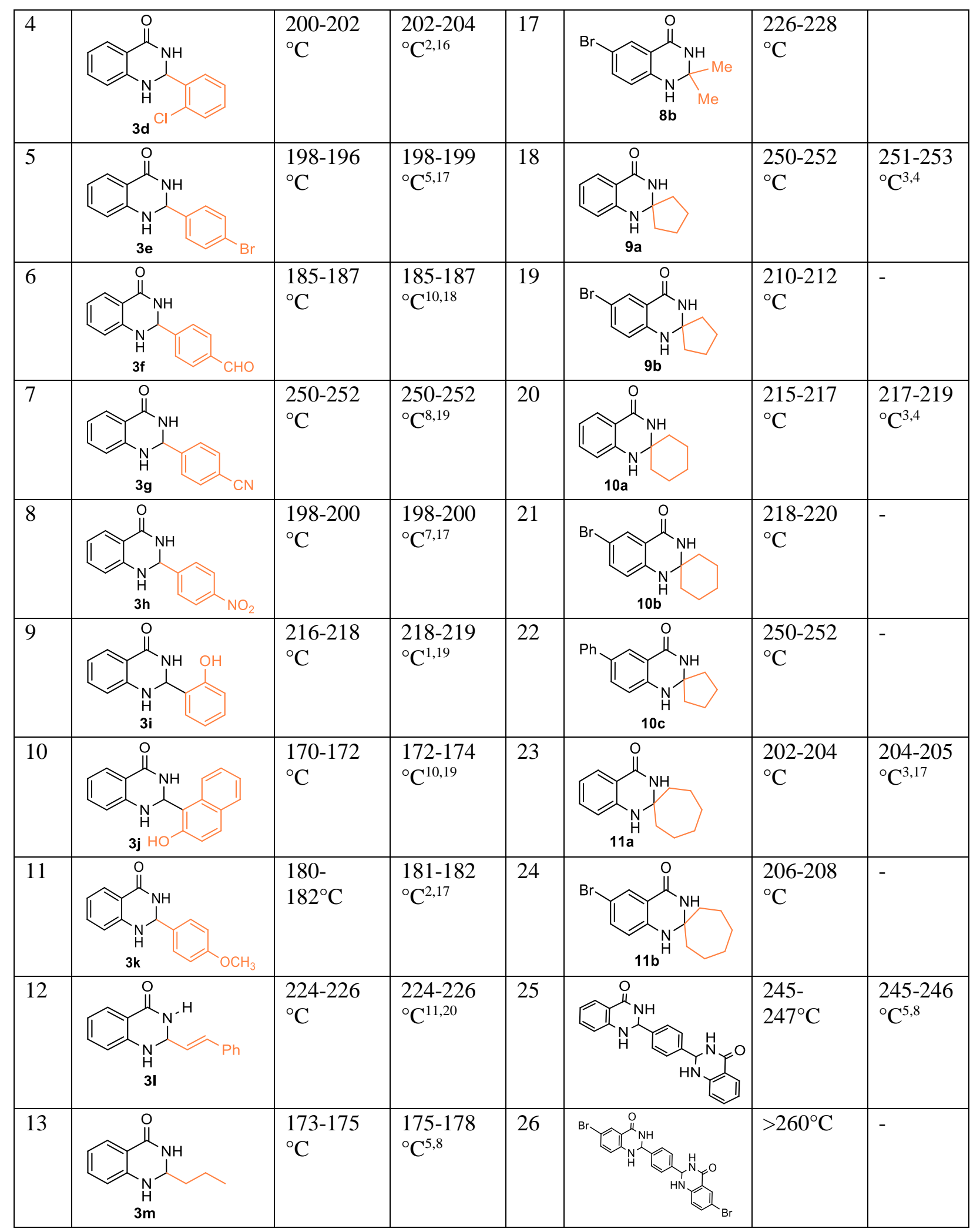


Characterization data of the 2,3-dihydroquinazolin-4(1H)-one derivatives

2-Phenyl-2,3-dihydroquinazolin-4(1H)-one (3a): ${ }^{9,13}$ Color = White solid $\quad(0.156$ g, $95 \%$ yield); MP $=220-222^{\circ} \mathrm{C}$ (purified by washing with PE/EA = 98:2); IR (KBr): 3305, 3180, 3059, 1665, 1615, 1535 , $1450 \mathrm{~cm}^{-1} ;{ }^{1} \mathrm{H}$ NMR (400 MHz, DMSO- $\left.d_{6}\right) \delta$ : (in ppm) $5.72(\mathrm{~s}, 1 \mathrm{H}), 6.76-6.57(\mathrm{~m}, 2 \mathrm{H}), 7.64-7.00$ $(\mathrm{m}, 8 \mathrm{H}), \quad 8.24(\mathrm{~s}, 1 \mathrm{H}) ;{ }^{13} \mathrm{C} \mathrm{NMR}\left(100 \mathrm{MHz}, \mathrm{DMSO}-d_{6}\right) \delta$ : (in ppm) 66.9, 114.8, 115.4, 117.5, 127.3, $127.8,128.8,128.9,133.7,142.1,148.3,164.0$.

2-(p-Tolyl)-2,3-dihydroquinazolin-4(1H)-one (3b): ${ }^{2,14}$ Color $=$ White solid (0.140 g, $80 \%$ yield); MP $=224-225^{\circ} \mathrm{C}$ (purified by washing with $\left.\mathrm{PE} / \mathrm{EA}=98: 2\right)$; IR $(\mathrm{KBr}): 3311,1657,1607,1508,1484,1383$ $\mathrm{cm}^{-1}$; ${ }^{1} \mathrm{H}$ NMR (400 MHz, DMSO- $\left.d_{6}\right) \delta$ : (in ppm) $2.30(\mathrm{~s}, 3 \mathrm{H}), 5.73(\mathrm{~s}, 1 \mathrm{H}), 6.77-6.66(\mathrm{~m}, 2 \mathrm{H}), 7.06$ (s, 1H), 7.27-7.19 (m, 3H), 7.39 (d, $J=7.6 \mathrm{~Hz}, 2 \mathrm{H}), 7.63(\mathrm{~d}, J=8.0 \mathrm{~Hz}, 1 \mathrm{H}), 8.24(\mathrm{~s}, 1 \mathrm{H}) ;{ }^{13} \mathrm{C} \mathrm{NMR}$ $\left(100 \mathrm{MHz}, \mathrm{DMSO}-d_{6}\right) \delta$ : (in ppm) 21.2, 66.9, 114.9, 115.5, 117.5, 127.3, 127.8, 129.3, 133.7, 138.2, 139.1, 148.4, 164.1.

2-(4-Isopropylphenyl)-2,3-dihydroquinazolin-4(1H)-one (3c): ${ }^{\mathbf{1 0}, 15}$ Color $=$ White solid $(0.141 \mathrm{~g}, 85 \%$ yield); $\mathrm{MP}=162-164{ }^{\circ} \mathrm{C}$ (purified by washing with $\left.\mathrm{PE} / \mathrm{EA}=98: 2\right) ;{ }^{1} \mathrm{H}$ NMR (400 MHz, DMSO- $\left.d_{6}\right) \delta$ : (in ppm) $1.16(\mathrm{~d}, J=6.9 \mathrm{~Hz}, 6 \mathrm{H}), 2.85(\mathrm{dt}, \mathrm{J}=13.7,6.8 \mathrm{~Hz}, 1 \mathrm{H}), 5.69(\mathrm{~s}, 1 \mathrm{H}), 5.69(\mathrm{~s}, 1 \mathrm{H}), 6.64(\mathrm{t}, J=$ $7.4 \mathrm{~Hz}, 1 \mathrm{H}), 6.70(\mathrm{~d}, \mathrm{~J}=8.0 \mathrm{~Hz}, 1 \mathrm{H}), 7.02(\mathrm{~s}, 1 \mathrm{H}), 7.27-7.17$ (m, 3H), 7.39 (d, J=8.1 Hz, 2H), 7.58 $(\mathrm{d}, \mathrm{J}=6.8 \mathrm{~Hz}, 1 \mathrm{H}), 8.19$ (s, $1 \mathrm{H}) ;{ }^{13} \mathrm{C}$ NMR (100 MHz, DMSO- $\left.d_{6}\right) \delta$ : (in ppm) 24.3, 33.7, 67.0, 114.8, $115.4,117.5,126.7,127.4,127.8,133.7,139.4,148.4,149.2,164.1$.

2-(2-Chlorophenyl)-2,3-dihydroquinazolin-4(1H)-one (3d): ${ }^{2,9}$ Color = Off white solid; $(0.171 \mathrm{~g}$, $90 \%) ; \mathrm{MP}=200-202{ }^{\circ} \mathrm{C}$ (purified by washing with PE/EA $\left.=98: 2\right) ;{ }^{1} \mathrm{H}$ NMR $\left(400 \mathrm{MHz}, \mathrm{DMSO}-d_{6}\right) \delta$ : (in ppm) $6.11(\mathrm{~s}, 1 \mathrm{H}), 6.72(\mathrm{dd}, J=16.3,7.5 \mathrm{~Hz}, 2 \mathrm{H}), 6.98(\mathrm{~s}, 1 \mathrm{H}), 7.22(\mathrm{~d}, J=6.9 \mathrm{~Hz}, 1 \mathrm{H}), 7.37$ (s, $2 \mathrm{H}), 7.46(\mathrm{~s}, 1 \mathrm{H}), 7.63(\mathrm{~d}, \mathrm{~J}=5.6 \mathrm{~Hz}, 2 \mathrm{H}), 8.18(\mathrm{~s}, 1 \mathrm{H}) ;{ }^{13} \mathrm{C}$ NMR $\left(100 \mathrm{MHz}, \mathrm{DMSO}-d_{6}\right) \delta$ : (in ppm) 64.1, 115.0, 115.1, 117.9, 127.8, 127.9, 129.2, 130.0, 130.7, 132.3, 133.9, 138.3, 148.1, 164.0.

2-(4-Bromophenyl)-2,3-dihydroquinazolin-4(1H)-one (3e): ${ }^{5,16}$ Color $=$ White solid $(0.207 \mathrm{~g}, 93 \%$ yield); $\mathrm{MP}=196-198{ }^{\circ} \mathrm{C}$ (purified by washing with $\left.\mathrm{PE} / \mathrm{EA}=98: 2\right) ;{ }^{1} \mathrm{H}$ NMR (400 MHz, DMSO- $\left.d_{6}\right) \delta$ : (in ppm) $5.76(\mathrm{~s}, 1 \mathrm{H}), 6.68(\mathrm{t}, J=7.5 \mathrm{~Hz}, 1 \mathrm{H}), 6.75(\mathrm{~d}, J=8.1 \mathrm{~Hz}, 1 \mathrm{H}), 7.15(\mathrm{~s}, 1 \mathrm{H}), 7.25$ (t, $J=7.7$ $\mathrm{Hz}, 1 \mathrm{H}), 7.44(\mathrm{~d}, J=8.4 \mathrm{~Hz}, 2 \mathrm{H}), 7.60(\mathrm{t}, J=7.4 \mathrm{~Hz}, 3 \mathrm{H}), 8.34(\mathrm{~s}, 1 \mathrm{H}) ;{ }^{13} \mathrm{C}$ NMR (100 MHz, DMSO$\left.d_{6}\right) \delta$ : (in ppm) 67.7, 116.4, 116.8, 119.2, 123.5, 129.3, 131.0, 133.1, 135.3, 143.0, 149.5, 165.4.

4-(4-Oxo-1,2,3,4-tetrahydroquinazolin-2-yl)benzaldehyde (3f): ${ }^{10,17}$ Color $=$ White solid $(0.148 \mathrm{~g}$, $80 \%$ yield); $\mathrm{MP}=185-187^{\circ} \mathrm{C}$ (purified by washing with PE/EA = 98:2); IR (KBr): 3296, 3190,3070, $1656,1606 \mathrm{~cm}^{-1}$; ${ }^{1} \mathrm{H}$ NMR $\left(\mathrm{CDCl}_{3}+\mathrm{DMSO}_{6} 300 \mathrm{MHz}\right) \delta$ : (in ppm) $5.87(\mathrm{~s}, 1 \mathrm{H}), 6.75-6.67(\mathrm{~m}, 2 \mathrm{H})$, 7.24-7.19 (m, 2H), 7.38 (s, 1H), 7.85-7.67 (m, 5H), 9.95 (s, 1H); ${ }^{13} \mathrm{C} \mathrm{NMR}\left(\mathrm{CDCl}_{3}, 75 \mathrm{MHz}\right) \delta$ : (in ppm) $66.3,114.9,115.3,117.8,127.9,130.0,133.9,136.6,147.9,148.7,163.8,193.2$. 
4-(4-Oxo-1,2,3,4-tetrahydroquinazolin-2-yl)benzonitrile (3g): ${ }^{8,18}$ Color $=$ Pale-yellow crystal (0.164 $\mathrm{g}, 90 \%$ yield); $\mathrm{MP}=250-252{ }^{\circ} \mathrm{C}$ (purified by washing with $\left.\mathrm{PE} / \mathrm{EA}=98: 2\right) ;{ }^{1} \mathrm{H} \mathrm{NMR}(400 \mathrm{MHz}, \mathrm{DMSO}-$ $\left.d_{6}\right) \delta$ : (in ppm) $5.83(\mathrm{~s}, 1 \mathrm{H}), 6.67$ (t, $\left.J=7.28 \mathrm{~Hz}, 1 \mathrm{H}\right), 6.73$ (d, $\left.J=8.04 \mathrm{~Hz}, 1 \mathrm{H}\right), 7.23(\mathrm{t}, J=7.28 \mathrm{~Hz}$, 1H), 7.26 (s, 1H), 7.58 (d, $J=7.52 \mathrm{~Hz}, 1 \mathrm{H}), 7.63(\mathrm{~d}, J=8.04 \mathrm{~Hz}, 2 \mathrm{H}), 7.84(\mathrm{~d}, J=8.28 \mathrm{~Hz}, 2 \mathrm{H}), 8.45$ (br s, $1 \mathrm{H}) ;{ }^{13} \mathrm{C}$ NMR (100 MHz, DMSO-d $\left.d_{6}\right) \delta$ : (in ppm) 65.9, 111.5, 114.9, 115.3, 117.9, 127.8, 128.1, 132.8, 134.0, 147.7, 147.8, 163.8 .

2-(4-Nitrophenyl)-2,3-dihydroquinazolin-4(1H)-one (3h): ${ }^{7,16}$ Color = Yellow solid (0.183 g, 93\% yield); $\mathrm{MP}=198-200{ }^{\circ} \mathrm{C}$ (purified by washing with $\left.\mathrm{PE} / \mathrm{EA}=98: 2\right) ;{ }^{1} \mathrm{H}$ NMR $\left(400 \mathrm{MHz}, \mathrm{DMSO}-d_{6}\right) \delta$ : (in ppm) $5.88(\mathrm{~s}, 1 \mathrm{H}), 6.74-6.64(\mathrm{~m}, 2 \mathrm{H}), 7.21-7.28(\mathrm{~m}, 2 \mathrm{H}), 7.58(\mathrm{~d}, J=4 \mathrm{~Hz}, 1 \mathrm{H}), 7.71$ (d, $J=8$ $\mathrm{Hz}, 2 \mathrm{H}), 8.22$ (d, $J=8 \mathrm{~Hz}, 2 \mathrm{H}), 8.47$ (s, $1 \mathrm{H}) ;{ }^{13} \mathrm{C}$ NMR (100 MHz, DMSO- $d_{6}$ ) $\delta$ : (in ppm) 65.7, 115.0, $115.3,117.9,124.0,127.8,128.4,134.0,147.7,147.8,149.7,163.7$.

2-(2-Hydroxyphenyl)2,3-dihydroquinazolin-4(1H)-one (3i): ${ }^{1,18}$ Color = White solid $(0.158 \mathrm{~g}, 90 \%$ yield); $\mathrm{MP}=216-218{ }^{\circ} \mathrm{C}$ (purified by washing with $\left.\mathrm{PE} / \mathrm{EA}=98: 2\right) ;{ }^{1} \mathrm{H}$ NMR $\left(400 \mathrm{MHz}, \mathrm{DMSO}-d_{6}\right) \delta$ : (in ppm) $5.98(\mathrm{~s}, 1 \mathrm{H}), 6.64(\mathrm{t}, J=7.52 \mathrm{~Hz}, 1 \mathrm{H}), 6.71(\mathrm{~s}, 1 \mathrm{H}), 6.75(\mathrm{t}, J=8.28 \mathrm{~Hz}, 1 \mathrm{H}), 6.78(\mathrm{~d}, J=7.52$ $\mathrm{Hz}, 1 \mathrm{H}), 6.83(\mathrm{~d}, \mathrm{~J}=8.04 \mathrm{~Hz}, 1 \mathrm{H}), 7.13(\mathrm{t}, J=6.76 \mathrm{~Hz}, 1 \mathrm{H}), 7.20(\mathrm{t}, J=7.04 \mathrm{~Hz}, 1 \mathrm{H}), 7.31(\mathrm{~d}, J=7.8$ $\mathrm{Hz}, 1 \mathrm{H}), 7.59$ (d, $J=7.76 \mathrm{~Hz}, 1 \mathrm{H}), 7.90(\mathrm{~s}, 1 \mathrm{H}), 9.83$ (s, 1H); ${ }^{13} \mathrm{C}$ NMR (100 MHz, DMSO-d $) \delta$ : (in ppm) 61.6, 115.0, 115.2, 115.8, 117.4, 119.2, 127.6, 127.7, 127.7, 129.7, 133.6, 148.5, 155.0, 164.4.

2-(2-Hydroxynaphthalen-1-yl)-2,3-dihydroquinazolin-4(1H)-one $(3 \mathrm{jj}):^{10,18}$ Color $=$ Orange solid (0.191 g, 90\% yield) ; $\mathrm{MP}=170-172{ }^{\circ} \mathrm{C}$ (purified by washing with PE/EA = 98:2); ${ }^{1} \mathrm{H} \mathrm{NMR}(400 \mathrm{MHz}$, $\left.\mathrm{CD}_{3} \mathrm{OD}\right) \delta$ : (in ppm) $6.82(\mathrm{~d}, J=9.28 \mathrm{~Hz}, 1 \mathrm{H}), 7.22-7.30(\mathrm{~m}, 2 \mathrm{H}), 7.45$ (t, $\left.J=8.28 \mathrm{~Hz}, 1 \mathrm{H}\right), 7.56$ (t, $J$ $=8.04 \mathrm{~Hz}, 1 \mathrm{H}), 7.61(\mathrm{~d}, J=7.76 \mathrm{~Hz}, 2 \mathrm{H}), 7.66(\mathrm{~d}, J=8.28 \mathrm{~Hz}, 1 \mathrm{H}), 7.75(\mathrm{~d}, J=9.28 \mathrm{~Hz}, 1 \mathrm{H}), 8.18(\mathrm{~d}$, $J=8.56 \mathrm{~Hz}, 1 \mathrm{H}), 9.35$ (s, $1 \mathrm{H}) ;{ }^{13} \mathrm{C}$ NMR (100 MHz, DMSO- $\left.d_{6}\right) \delta$ : (in ppm) 109.2, 119.5, 120.7, 123.5, 123.9, 126.0, 127.0, 128.6, 128.8, 129.4, 129.5, 131.6, 134.0, 137.8, 142.4, 154.2, 169.6, 172.6.

2-(4-Methoxyphenyl)-2,3-dihydroquinazolin-4(1H)-one (3k):2,16 Color = White solid (0.112 g, 60\% yield); $\mathrm{MP}=180-182{ }^{\circ} \mathrm{C}$ (purified by washing with $\left.\mathrm{PE} / \mathrm{EA}=98: 2\right) ;{ }^{1} \mathrm{H}$ NMR (400 MHz, DMSO- $\left.d_{6}\right) \delta$ : (in ppm) $3.75(\mathrm{~s}, 3 \mathrm{H}), 5.72(\mathrm{~s}, 1 \mathrm{H}), 6.68(\mathrm{~s}, 1 \mathrm{H}), 6.75(\mathrm{~d}, J=8.1 \mathrm{~Hz}, 1 \mathrm{H}), 6.96(\mathrm{~s}, 2 \mathrm{H}), 7.02(\mathrm{~s}, 1 \mathrm{H})$, $7.25(\mathrm{~s}, 1 \mathrm{H}), 7.43(\mathrm{~d}, J=8.6 \mathrm{~Hz}, 2 \mathrm{H}), 7.62(\mathrm{~d}, J=7.6 \mathrm{~Hz}, 1 \mathrm{H}), 8.19(\mathrm{~s}, 1 \mathrm{H}) ;{ }^{13} \mathrm{C}$ NMR $(100 \mathrm{MHz}, \mathrm{DMSO}-$ $\left.d_{6}\right) \delta$ : (in ppm) 57.1, 68.2, 115.5, 116.3, 116.9, 119.0,129.2, 130.1, 135.1, 135.4, 149.9, 161.3, 165.6.

(E)-2-Styryl-2,3-dihydroquinazolin-4(1H)-one (31): ${ }^{11.19}$ Color = Pale yellow solid $(0.091 \mathrm{~g}, 50 \%$ yield) $; \mathrm{MP}=224-226{ }^{\circ} \mathrm{C}$ (purified by washing with $\left.\mathrm{PE} / \mathrm{EA}=98: 2\right) ;{ }^{1} \mathrm{H}$ NMR (500 MHz, DMSO-d $)$ $\delta$ : (in ppm) $5.31(\mathrm{~d}, J=6.7 \mathrm{~Hz}, 1 \mathrm{H}), 6.37(\mathrm{dd}, J=15.8 \mathrm{~Hz}$ and $6.8 \mathrm{~Hz}, 1 \mathrm{H}), 6.67(\mathrm{dd}, J=14.9 \mathrm{~Hz}$ and $6.5 \mathrm{~Hz}, 2 \mathrm{H}), 6.75$ (d, $J=8.0 \mathrm{~Hz}, 1 \mathrm{H}), 6.89$ (br s, 1H), 7.29-7.23 (m, 2H), 7.34 (t, $J=7.5 \mathrm{~Hz}, 2 \mathrm{H}), 7.45$ (d, $J=7.5 \mathrm{~Hz}, 2 \mathrm{H}), 7.62(\mathrm{~d}, J=7.6 \mathrm{~Hz}, 1 \mathrm{H}), 8.14$ (brs, $1 \mathrm{H}) ;{ }^{13} \mathrm{C}$ NMR $\left(125 \mathrm{MHz}, \mathrm{DMSO}-d_{6}\right) \delta$ : (in ppm) $65.8,114.5,114.8,117.1,126.6,127.3,128.1,128.3,128.7,131.6,133.2,135.7,147.7,163.3$. 
2-Propyl-2,3-dihydroquinazolin-4(1H)-one (3m): ${ }^{\mathbf{5}, 8}$ Color $=$ Red solid $(0.125 \mathrm{~g}, 90 \%$ yield $)$; $\mathrm{MP}=$ $173-175{ }^{\circ} \mathrm{C}$ (purified by washing with PE/EA = 98:2); IR (KBr): 3214, 3021, $1670 \mathrm{~cm}^{-1} ;{ }^{1} \mathrm{H}$ NMR (400 $\left.\mathrm{MHz}, \mathrm{CDCl}_{3}\right) \delta$ : (in ppm) $1.01(\mathrm{t}, J=4.0 \mathrm{~Hz}, 3 \mathrm{H}), 1.52-1.46(\mathrm{~m}, 2 \mathrm{H}), 1.78(\mathrm{q}, 2 \mathrm{H}), 4.22$ (brs, $\left.1 \mathrm{H}\right), 4.90$ $(\mathrm{t}, J=4.0 \mathrm{~Hz}, 1 \mathrm{H}), 6.24(\mathrm{brs}, 1 \mathrm{H}), 6.67(\mathrm{~d}, J=4.0 \mathrm{~Hz}, 1 \mathrm{H}), 6.87(\mathrm{t}, J=6.0 \mathrm{~Hz}, 1 \mathrm{H}), 7.31(\mathrm{t}, J=4.0 \mathrm{~Hz}$, $1 \mathrm{H}), 7.89(\mathrm{~d}, J=4.0 \mathrm{~Hz}, 1 \mathrm{H}) ;{ }^{13} \mathrm{C} \mathrm{NMR}\left(\mathrm{CDCl}_{3}, 100 \mathrm{MHz}\right) \delta$ : (in ppm) 14.0, 17.6, 37.8, 65.3, 114.9, 116.1, 119.6, 128.8, 134.1, 147.6, 165.7 .

2-Cyclohexyl-2,3-dihydroquinazolin-4(1H)-one (3n): ${ }^{9,16}$ Color $=$ White solid (0.135 g, $80 \%$ yield); $\mathrm{MP}=178-180{ }^{\circ} \mathrm{C}$ (purified by washing with $\left.\mathrm{PE} / \mathrm{EA}=98: 2\right) ;{ }^{1} \mathrm{H} \mathrm{NMR}\left(400 \mathrm{MHz}, \mathrm{DMSO}-d_{6}\right) \delta$ : (in ppm) $1.11(\mathrm{~s}, 5 \mathrm{H}), 1.70(\mathrm{~d}, J=10.6 \mathrm{~Hz}, 6 \mathrm{H}), 4.44(\mathrm{~s}, 1 \mathrm{H}), 6.54(\mathrm{~s}, 1 \mathrm{H}), 6.60(\mathrm{~s}, 1 \mathrm{H}), 6.74(\mathrm{~d}, J=8.1 \mathrm{~Hz}, 1 \mathrm{H})$, $7.19(\mathrm{~s}, 1 \mathrm{H}), 7.55(\mathrm{~d}, J=7.6 \mathrm{~Hz}, 1 \mathrm{H}), 7.87(\mathrm{~s}, 1 \mathrm{H}) ;{ }^{13} \mathrm{C}$ NMR (100 MHz, DMSO-d $\left.d_{6}\right) \delta$ : (in ppm) 27.4, 27.5, 27.8, 28.6, 28.9, 41.2, 41.4, 41.6, 44.7, 70.5, 116.0, 116.7, 118.3, 129.1, 134.9, 150.2, 165.6.

2-(Thiophen-2-yl)-2,3-dihydroquinazolin-4(1H)-one (3o): ${ }^{2,14}$ Color $=$ White Solid $(0.143 \mathrm{~g}, 85 \%$ yield); $\mathrm{MP}=223-224^{\circ} \mathrm{C}$ (purified by washing with $\mathrm{PE} / \mathrm{EA}=98: 2$ ); IR $(\mathrm{KBr}): 3287,1650,1607,1515$, 1487, $763 \mathrm{~cm}-1$; ${ }^{1} \mathrm{H}$ NMR (400 MHz, DMSO- $\left.d_{6}\right) \delta$ : (in ppm) 6.03 (s, 1H), $6.79-6.70$ (m, 2H), 6.99 (t, $J=3.6 \mathrm{~Hz}, 1 \mathrm{H}), 7.14(\mathrm{~d}, J=2.8 \mathrm{~Hz}, 1 \mathrm{H}), 7.29-7.25(\mathrm{~m}, 2 \mathrm{H}), 7.46$ (d, $J=4.8 \mathrm{~Hz}, 1 \mathrm{H}), 7.64$ (d, $J=8.0$ $\mathrm{Hz}, 1 \mathrm{H}), 8.46(\mathrm{~s}, 1 \mathrm{H}) ;{ }^{13} \mathrm{C}$ NMR (100 MHz, DMSO- $\left.d_{6}\right) \delta$ : (in ppm) 63.0, 115.2, 115.6, 118.0, 126.2, $126.4,126.9,127.8,133.8,146.9,147.7,163.6$.

2,2-Dimethyl-2,3-dihydroquinazolin-4(1H)-one (8a): ${ }^{4.20}$ Color $=$ white solid $(0.110$ g, $85 \%$ yield); $\mathrm{MP}=182-184{ }^{\circ} \mathrm{C}$ (purified by crystallization in methanol); IR (KBr): 3255, 1632, 1485, 1270, 1175 , $750 \mathrm{~cm}^{-1}$; ${ }^{1} \mathrm{H}$ NMR $\left(400 \mathrm{MHz}, \mathrm{CDCl}_{3}\right) \delta$ : (in ppm) 1.58 (s, 6H), 4.25 (br s, $\left.1 \mathrm{H}\right), 6.64$ (d, J = $7.6 \mathrm{~Hz}$, $1 \mathrm{H}), 6.83(\mathrm{t}, J=7.2 \mathrm{~Hz}, 1 \mathrm{H}), 7.05(\mathrm{brs}, 1 \mathrm{H}), 7.31(\mathrm{t}, J=8.0 \mathrm{~Hz}, 1 \mathrm{H}), 7.89(\mathrm{~d}, J=7.2 \mathrm{~Hz}, 1 \mathrm{H}) ;{ }^{13} \mathrm{C} \mathrm{NMR}$ $\left(100 \mathrm{MHz}, \mathrm{CDCl}_{3}\right) \delta$ : (in ppm) 29.6, 67.6, 114.6, 114.7, 118.7, 128.3, 133.9, 146.0, 164.7.

1'H-Spiro[cyclopentane-1,2'-quinazolin]-4'(3'H)-one (9a): ${ }^{3,4}$ Color = White solid $(0.133 \mathrm{~g}, 90 \%$ yield); $\mathrm{MP}=250-252{ }^{\circ} \mathrm{C}$ (purified by crystallization in methanol); IR (KBr):3292, 3163, 1639,1616 $\mathrm{cm}^{-1}$; ${ }^{1} \mathrm{H}$ NMR (400 MHz, DMSO-d $d_{6} \delta$ : (in ppm) 1.64-1.68 (m, $\left.4 \mathrm{H}\right), 1.77-1.79(\mathrm{~m}, 4 \mathrm{H}), 6.63(\mathrm{t}, J=$ $7.6 \mathrm{~Hz}, 1 \mathrm{H}), 6.70(\mathrm{~d}, J=8.0 \mathrm{~Hz}, 1 \mathrm{H}), 6.74(\mathrm{~s}, 1 \mathrm{H}), 7.21(\mathrm{t}, J=8.4 \mathrm{~Hz}, 1 \mathrm{H}), 7.57(\mathrm{~d}, J=8.0 \mathrm{~Hz}, 1 \mathrm{H})$, 8.10 (s, 1H); ${ }^{13} \mathrm{C}$ NMR (100 MHz, DMSO-d $\left.d_{6}\right) \delta$ (in ppm) 22.4, 77.5, 114.8, 115.0, 117.0, 127.7, 133.4, 147.9, 163.9.

1'H-Spiro[cyclohexane-1,2'-quinazolin]-4'(3'H)-one (10a): ${ }^{3,4}$ Color = White solid $(0.142$ g, 90\% yield); $\mathrm{MP}=215-217^{\circ} \mathrm{C}$ (purified by crystallization in methanol); IR (KBr): 1614, 1643, 3183, 3286 $\mathrm{cm}^{-1} .{ }^{1} \mathrm{H}$ NMR (400 MHz, DMSO-d $) \delta$ : (in ppm) $1.22(\mathrm{~s}, 1 \mathrm{H}), 1.38(\mathrm{~s}, 1 \mathrm{H}), 1.53$ (br s, 6H), 1.70 (s, 2H), 6.56 (br s, 2H), 6.77 (d, $J=4 \mathrm{~Hz}, 1 \mathrm{H}), 7.17$ (br s, $1 \mathrm{H}), 7.52(\mathrm{~d}, J=5.7 \mathrm{~Hz}, 1 \mathrm{H}), 7.86(\mathrm{~s}, 1 \mathrm{H}) ;{ }^{13} \mathrm{C}$ NMR (100 MHz, DMSO- $\left.d_{6}\right) \delta$ : (in ppm) 21.3, 25.1, 37.6, 68.2, 114.9, 115.0, 116.9, 127.5, 133.5, 147.2, 163.6 . 
1'H-spiro[cycloheptane-1,2'-quinazolin]-4'(3'H)-one (11a): ${ }^{3,16}$ Color = Off white solid (0.143 g, $85 \%$ yield); $\mathrm{MP}=202-204{ }^{\circ} \mathrm{C}$ (purified by crystallization in methanol); ${ }^{1} \mathrm{H}$ NMR (400 MHz, DMSO$\left.d_{6}\right) \delta$ : (in ppm) $1.50(\mathrm{~s}, 8 \mathrm{H}), 1.87(\mathrm{~s}, 4 \mathrm{H}), 6.59(\mathrm{~s}, 1 \mathrm{H}), 6.71(\mathrm{~s}, 2 \mathrm{H}), 7.20(\mathrm{t}, J=7.6 \mathrm{~Hz}, 1 \mathrm{H}), 7.54(\mathrm{~d}, J$ $=7.7 \mathrm{~Hz}, 1 \mathrm{H}), 8.02(\mathrm{~s}, 1 \mathrm{H}) ;{ }^{13} \mathrm{C}$ NMR $\left(100 \mathrm{MHz}, \mathrm{DMSO}-d_{6}\right) \delta$ : (in ppm) 22.7, 31.1, 42.9, 73.8, 116.1, $116.2,118.2,135.0,148.6,164.5$.

2,2'-(1,4-Phenylene)bis(2,3-dihydroquinazol-4(1H)-one) (13a): ${ }^{5,8}$ Color = Light brown solid (0.234 $\mathrm{g}, 85 \%$ yield); $\mathrm{MP}=245-247^{\circ} \mathrm{C}$ (purified by silica-gel column chromatography using DCM/Methanol = 9.3:0.7); IR (KBr): 3416, 3212, 1663, $1669 \mathrm{~cm}-1 ;{ }^{1} \mathrm{H} \mathrm{NMR}\left(\mathrm{CDCl}_{3}, 400 \mathrm{MHz}\right): \delta 5.89$ (s, $\left.1 \mathrm{H}\right), 5.96$ (s, 1H), $5.96(\mathrm{~s}, 1 \mathrm{H}), 6.77(\mathrm{~d}, J=8.0 \mathrm{~Hz}, 1 \mathrm{H}), 6.85(\mathrm{t}, J=8.0 \mathrm{~Hz}, 2 \mathrm{H}), 6.91(\mathrm{br} \mathrm{s}, 1 \mathrm{H}), 7.32$ (t, $J=6.0$ $\mathrm{Hz}, 2 \mathrm{H}), 7.43$ (s, 2H), 7.62 (s, 1H), 7.78(d, $J=8.0 \mathrm{~Hz}, 2 \mathrm{H}), 7.87$ (d, $J=8.0 \mathrm{~Hz}, 2 \mathrm{H}), 7.94$ ( d, $J=8.0$ $\mathrm{Hz}, 2 \mathrm{H}), 10.04(\mathrm{~s}, 1 \mathrm{H}) ;{ }^{13} \mathrm{C} \mathrm{NMR}\left(\mathrm{CDCl}_{3}+\mathrm{DMSO}-d_{6}, 100 \mathrm{MHz}\right): \delta=66.3,114.2,114.7,117.1,126.6$, 127.2, 129.3, 133.0, 141.6, 147.4, 164.0.

Table S2. Reproducibility of the reaction between anthranilamide 1a and benzaldehyde 2a

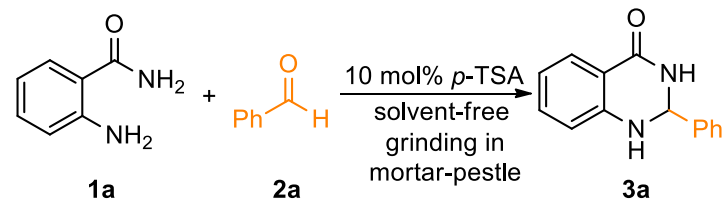

\begin{tabular}{|c|c|c|}
\hline $\begin{array}{c}\text { Sl. } \\
\text { No. }\end{array}$ & $\begin{array}{c}\text { Time required for complete } \\
\text { conversion of } \mathbf{1 a}\end{array}$ & \% yield \\
\hline 1 & 3 & 95 \\
\hline 2 & 3 & 94 \\
\hline 3 & 3 & 95 \\
\hline
\end{tabular}

Solubility profile of anthranilamide and benzaldehyde in molten $\boldsymbol{p}$-TSA

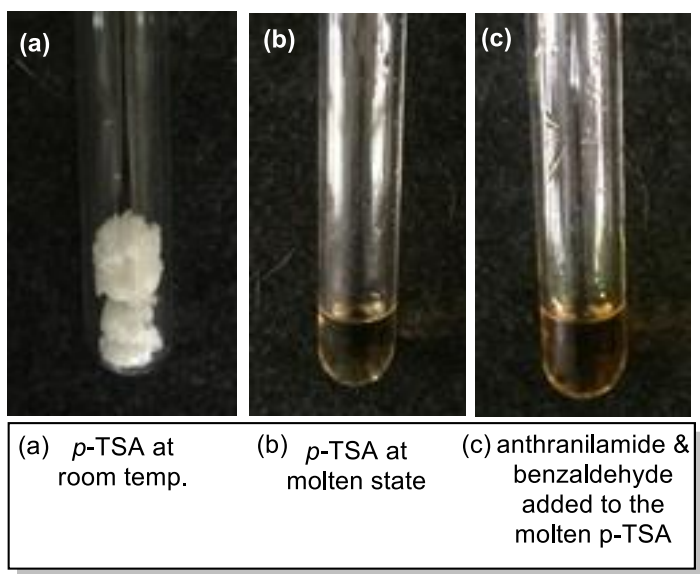

Figure S1. Solubility profile of anthranilamide and benzaldehyde in molten $p$-TSA. 
Calculation Of The Green Chemistry Metrics for the reaction between anthranilamide 1a and $p$ nitrobenzaldehyde $2 \mathrm{~h}$ in presence of $10 \mathrm{~mol} \% \mathrm{p}$-TSA

Scheme S3. Synthesis of 2-(4-nitrophenyl)-2,3-dihydroquinazolin-4(1H)-one (3h) from anthranilamide and $p$-nitrobenzaldehyde by grinding in mortar pestle

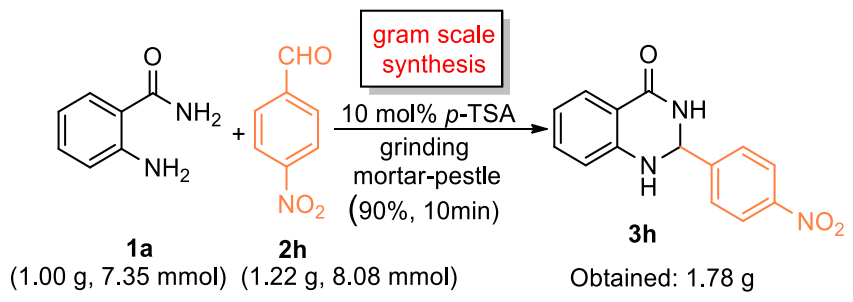

Table S3. Calculation Of The Green Chemistry Metrics.

\begin{tabular}{|c|c|c|}
\hline 1 & Atom economy ${ }^{[12]}$ & \\
\hline & $\begin{array}{l}\text { Atom Economy }=(\text { Exact molecular mass of } \\
\text { desired product }(\mathrm{C}) / \text { Exact Molecular mass } \\
\text { of all reactant }(\mathrm{A}+\mathrm{B})) \text { X } 100 \%\end{array}$ & $\begin{array}{l}=(269.26 / 136.15+1.1(151.12) \times 100 \% \\
\mathrm{AE}=\mathbf{8 9 . 0 4 \%}\end{array}$ \\
\hline 2 & Reaction mass efficiency ${ }^{[12]}$ & \\
\hline & $\begin{array}{l}\text { Reaction Mass Efficiency = (Mass of product } \\
\text { (C)/ Mass of reactant (A) + Mass of reactant } \\
\text { (B)) X } 100\end{array}$ & $\begin{array}{l}=(1.786 /(1+1.220)) \times 100 \\
\mathrm{RME}=\mathbf{8 0 . 4 5 \%}\end{array}$ \\
\hline 3 & E-factor ${ }^{[12]}$ & \\
\hline & $\begin{array}{l}\text { E-Factor = Amount of waste } / \text { Amount of } \\
\text { product }\end{array}$ & $\begin{array}{l}\text { Total amount of reactant }=\mathrm{A}+\mathrm{B}=(1.0+ \\
1.220) \mathrm{g}=2.220 \mathrm{~g} \\
\text { Amount of final product }=1.786 \mathrm{~g} \\
\text { Amount of waste }=(\text { Total amount of reactant } \\
- \text { amount of final product }) \\
=(2.220-1.786) \mathrm{g} \\
=0.434 \mathrm{~g} \\
\mathrm{E} \text {-factor }=(0.434 / 1.786)=\mathbf{0 . 2 4 3 0}\end{array}$ \\
\hline 4 & Ecoscale $^{[12]}$ & \\
\hline & $\begin{array}{l}\text { Ecoscale }=(100-\text { Sum of individual } \\
\text { penalties })\end{array}$ & $\begin{array}{l}\text { Eco-scale }=100-18.07 \\
=\mathbf{8 1 . 9 3}\end{array}$ \\
\hline
\end{tabular}

Table S4. Calculation of the penalty points for the ecoscale calculation.

\begin{tabular}{|l|l|l|}
\hline \multicolumn{2}{|l|}{ Calculation of penalty points } & Penalty points \\
\hline $\begin{array}{l}\text { Sr. } \\
\text { No }\end{array}$ & Parameters & \\
\hline 1 & Reaction Yield & 5.07 \\
\hline & Yield $=89 \%$ & \\
\hline 2 & $\begin{array}{l}\text { Price of reaction components (to obtain 10mmol of final } \\
\text { product) }\end{array}$ & \\
\hline
\end{tabular}




\begin{tabular}{|l|l|l|}
\hline & Anthranilamide & 3 \\
\hline & 4 -Nitro Benzaldehyde & 0 \\
\hline & $p$-TSA & 0 \\
\hline 3 & Safety & \\
\hline & Anthranilamide & 5 \\
\hline & 4 -nitro Benzaldehyde & 5 \\
\hline & p-TSA & 0 \\
\hline 4 & Technical setup & \\
\hline & Common setup & 0 \\
\hline 5 & Temperature/Time & \\
\hline & Room temperature & 0 \\
\hline 6 & Work and Purification & \\
\hline & Water wash and Simple filtration & 0 \\
\hline 7 & Total penalty points & 18.07 \\
\hline
\end{tabular}

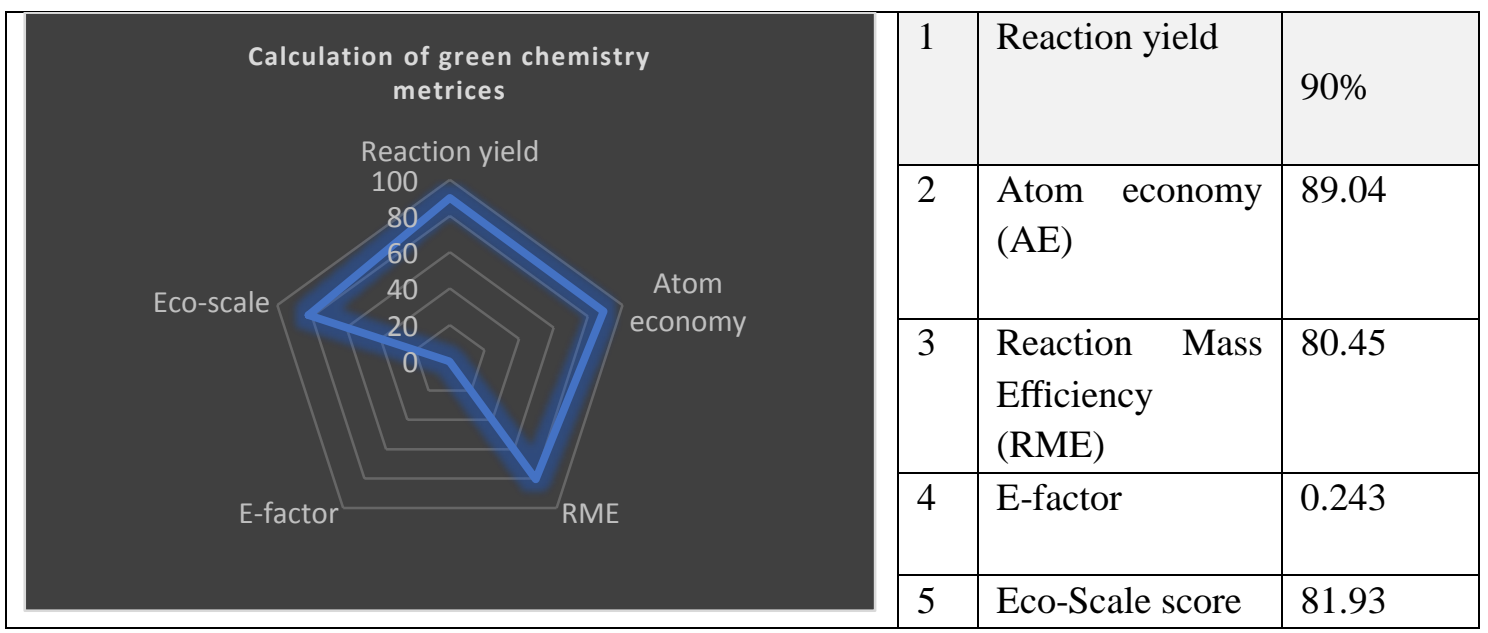

Figure S2. Green chemistry metrices for the synthesis of 2-(4-nitrophenyl)-2,3-dihydroquinazolin$4(1 \mathrm{H})$-one $(\mathbf{3 h})$ from anthranilamide and $p$-nitrobenzaldehyde by grinding in mortar pestle.

Process Intensification: Mechanochemical scalable synthesis of 2-(4-nitrophenyl)-2,3dihydroquinazolin-4(1H)-one (3h) by ball milling

Ball milling was carried out in tumbler ball milling instrument purchased from S.F. Engineering works having frequency $40 \mathrm{rpm}$, voltage $230 \mathrm{~V}$ and motor power $18.5 \mathrm{~kW}$ and current $24 \mathrm{~A}$. The internal diameter of milling vessel was $5 \mathrm{~cm}$ with $200 \mathrm{ml}$ capacity. 


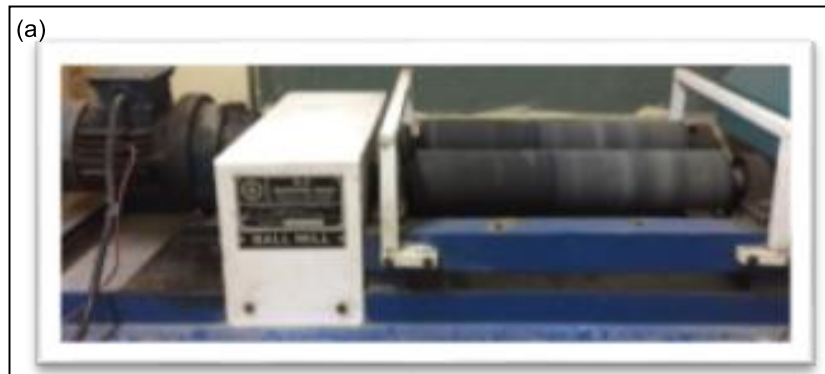

(b)

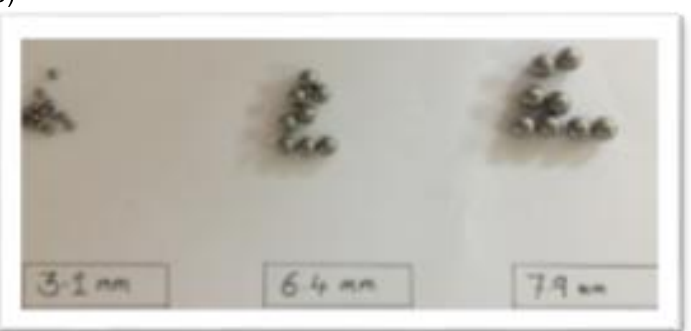

Figure S3. (a) Tumbler ball mill; (b) Milling balls of variable sizes.

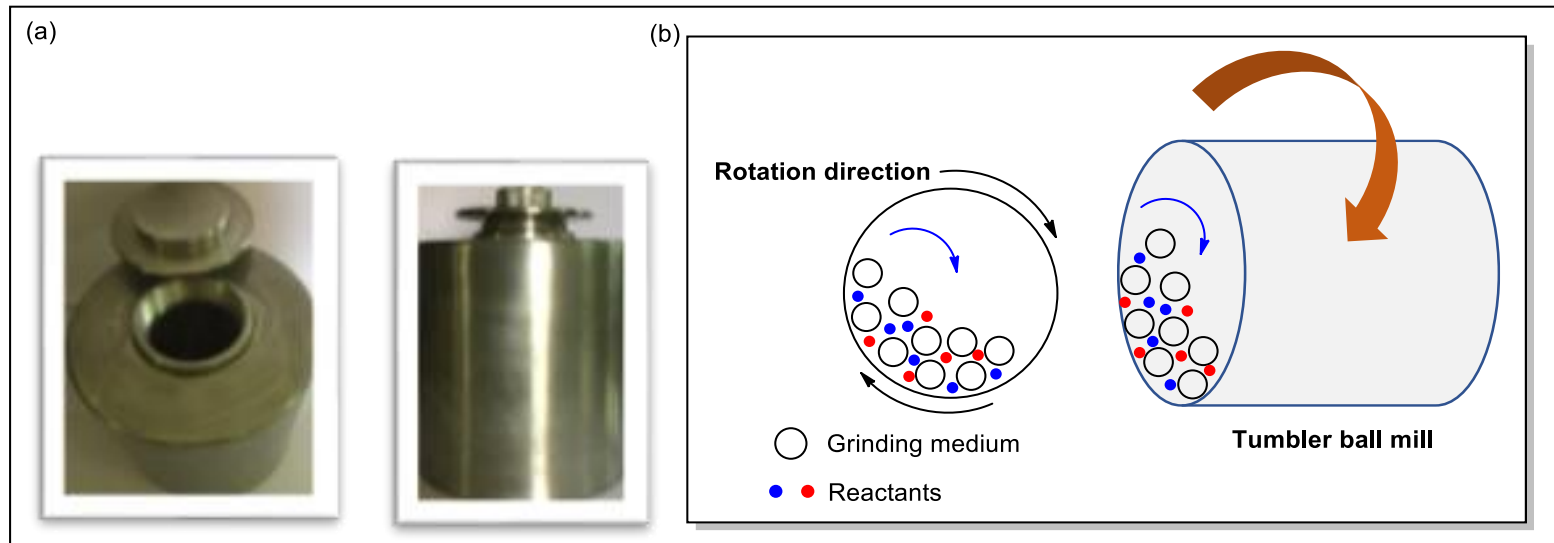

Figure S4. (a) Ball milling vessel; (b) Representation of mechanical ball milling operation in tumbler ball mill.

Scheme S4. Multigram synthesis of 2-(4-nitrophenyl)-2,3-dihydroquinazolin-4(1H)-one (3h) by mechanochemical ball milling.

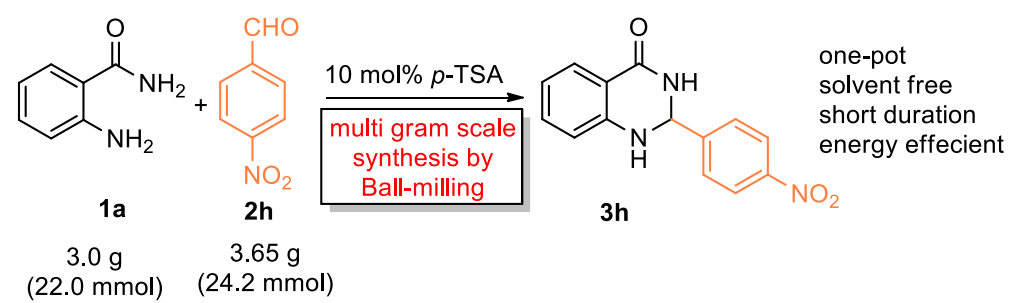

Procedure: To a mixture of anthranilamide $1 \mathrm{a}(0.1 \mathrm{~g}, 0.73 \mathrm{mmol}, 1$ equiv. $)$ and $p$-nitrobenzaldehyde 2h $(0.122 \mathrm{~g}, 0.81 \mathrm{mmol}, 1.1$ equiv.) was added $10 \mathrm{~mol} \%$ of $p$-TSA catalyst and the reaction mixture was milled in a ball mill consisting of stainless-steel balls. The progress of the reaction was montiored by TLC and HPLC analyses and on completion of the reaction, water was added, and the product was filtered off and dried to get hold of the desired product in excellent yield and purity.

Similar ball milling experiments were subsequently carried out with different ball diameters.

Table S5. Mechanochemical synthesis of 2-(4-nitrophenyl)-2,3-dihydroquinazolin-4(1H)-one (3h) by ball milling under several conditions. 


\begin{tabular}{|c|c|c|c|c|c|}
\hline \multirow[t]{2}{*}{$\begin{array}{l}\text { Sl. } \\
\text { No. }\end{array}$} & \multirow[t]{2}{*}{$\begin{array}{l}\text { Time } \\
\text { (mins) }\end{array}$} & \multicolumn{4}{|c|}{$\begin{array}{l}\text { Conversion of anthranilamide 1a under } \\
\text { different milling conditions }{ }^{b}\end{array}$} \\
\hline & & $\begin{array}{l}\text { Condition } \\
\mathrm{A}^{\mathrm{c}, \mathrm{d}}\end{array}$ & $\begin{array}{l}\text { Condition } \\
\mathrm{B}^{\mathrm{c}, \mathrm{d}}\end{array}$ & $\begin{array}{l}\text { Condition } \\
\mathrm{C}^{\mathrm{c}, \mathrm{d}}\end{array}$ & $\begin{array}{l}\text { Condition } \\
\mathrm{D}^{\mathrm{c}, \mathrm{d}}\end{array}$ \\
\hline 1 & 10 & 60 & 30 & 70 & 55 \\
\hline 2 & 20 & 65 & 50 & 75 & 60 \\
\hline 3 & 30 & 70 & 55 & 80 & 70 \\
\hline 4 & 40 & 75 & 65 & 85 & 75 \\
\hline 5 & 50 & 78 & 70 & 87 & 80 \\
\hline 6 & 60 & 83 & 75 & 90 & 85 \\
\hline 7 & 120 & 86 & 79 & 93 & 90 \\
\hline 8 & 240 & 90 & 83 & 98 & 93 \\
\hline
\end{tabular}

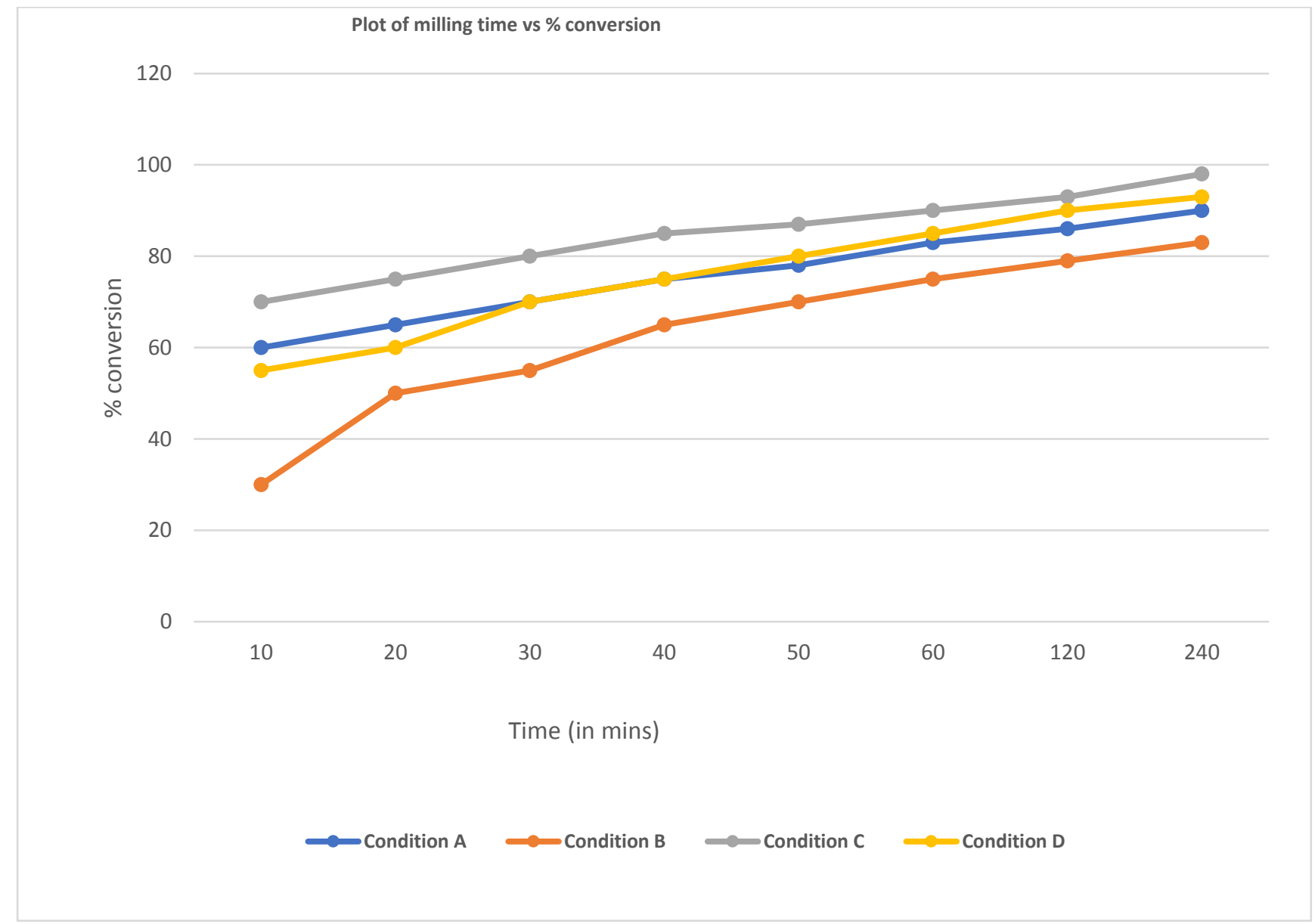

Figure S5. Graphical plot of the conversion of anthranilamide vs time interval (in mins) during the mechanochemical milling process. 
${ }^{1} \mathrm{H}$ and ${ }^{13} \mathrm{C}$ NMR Spectral Reproductions Of Representative 2,3-Dihydroquinazolin-4(1H)-one (3a, 3c, 3d, 3h, 8b, 9b, 10a, 10b, 10c, 11b and 13b)
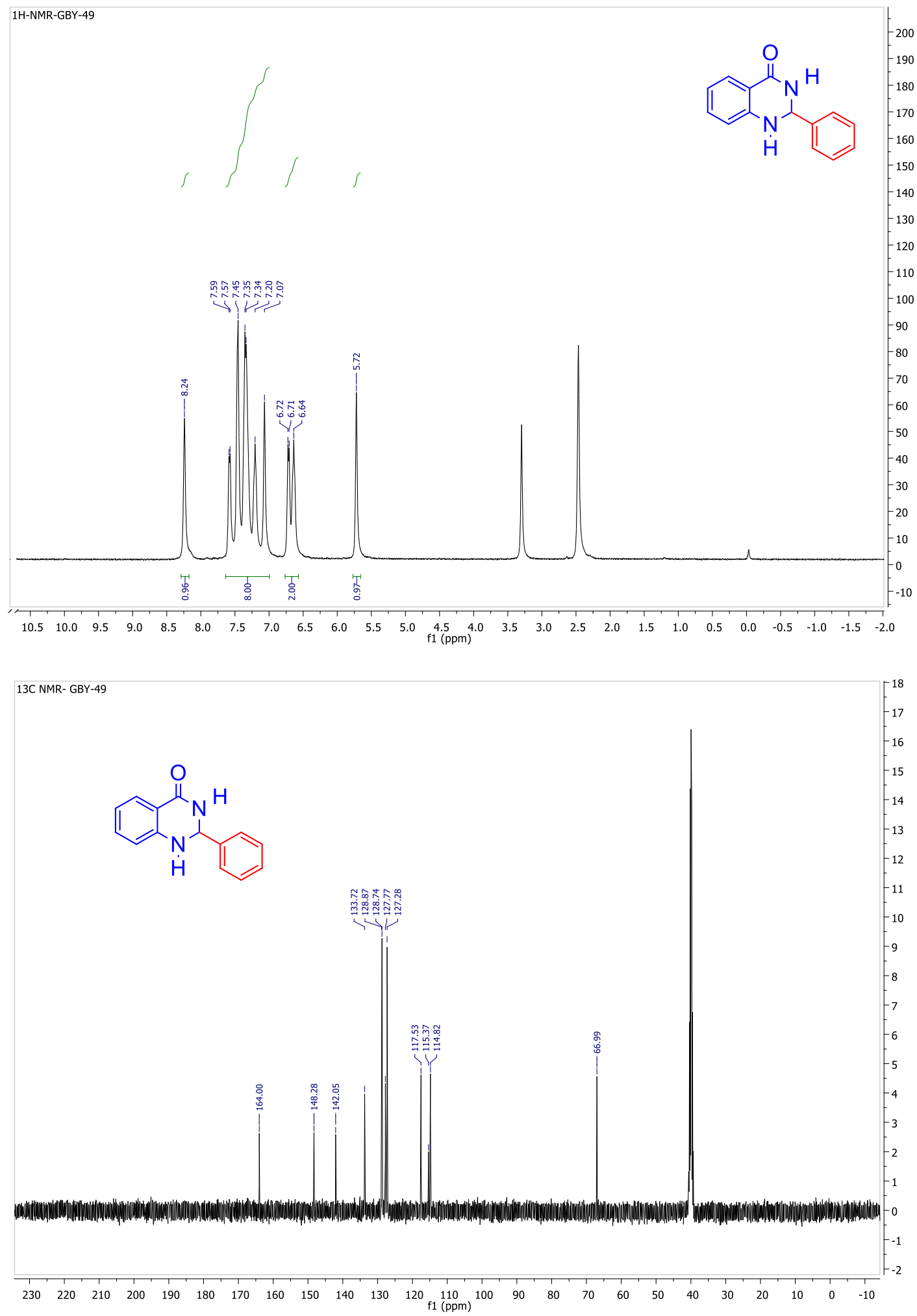

Figure S6. ${ }^{1} \mathrm{H}\left(400 \mathrm{MHz}, \mathrm{DMSO}-d_{6}\right)$ and ${ }^{13} \mathrm{C}$ NMR $\left(100 \mathrm{MHz}, \mathrm{DMSO}-d_{6}\right)$ spectral reproduction of 2phenyl-2,3-dihydroquinazolin-4(1H)-one (3a). 

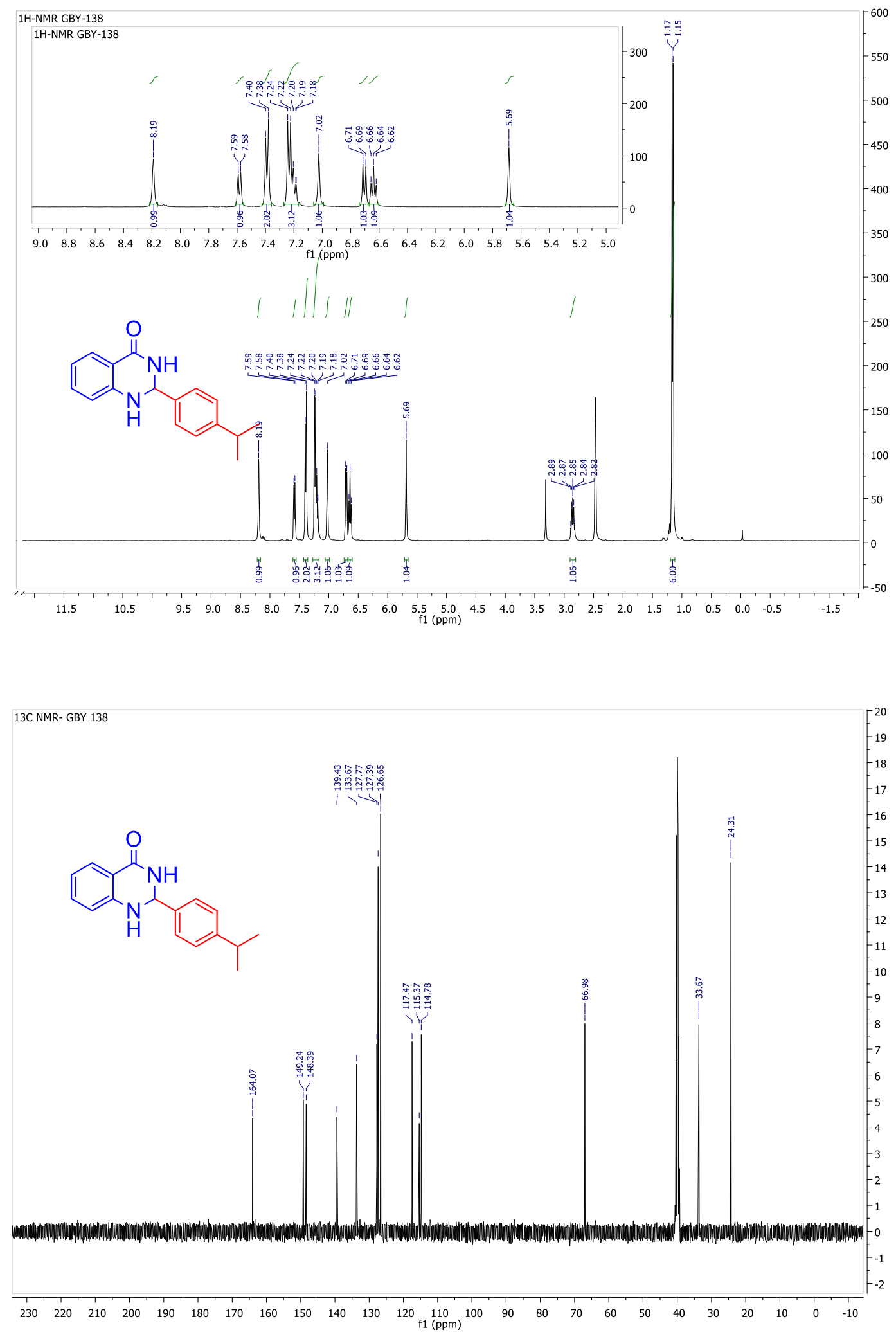

Figure S7. ${ }^{1} \mathrm{H}\left(400 \mathrm{MHz}, \mathrm{DMSO}-d_{6}\right)$ and ${ }^{13} \mathrm{C}$ NMR $\left(100 \mathrm{MHz}, \mathrm{DMSO}-d_{6}\right)$ spectral reproduction of 2(4-isopropylphenyl)-2,3-dihydroquinazolin-4(1H)-one (3c). 

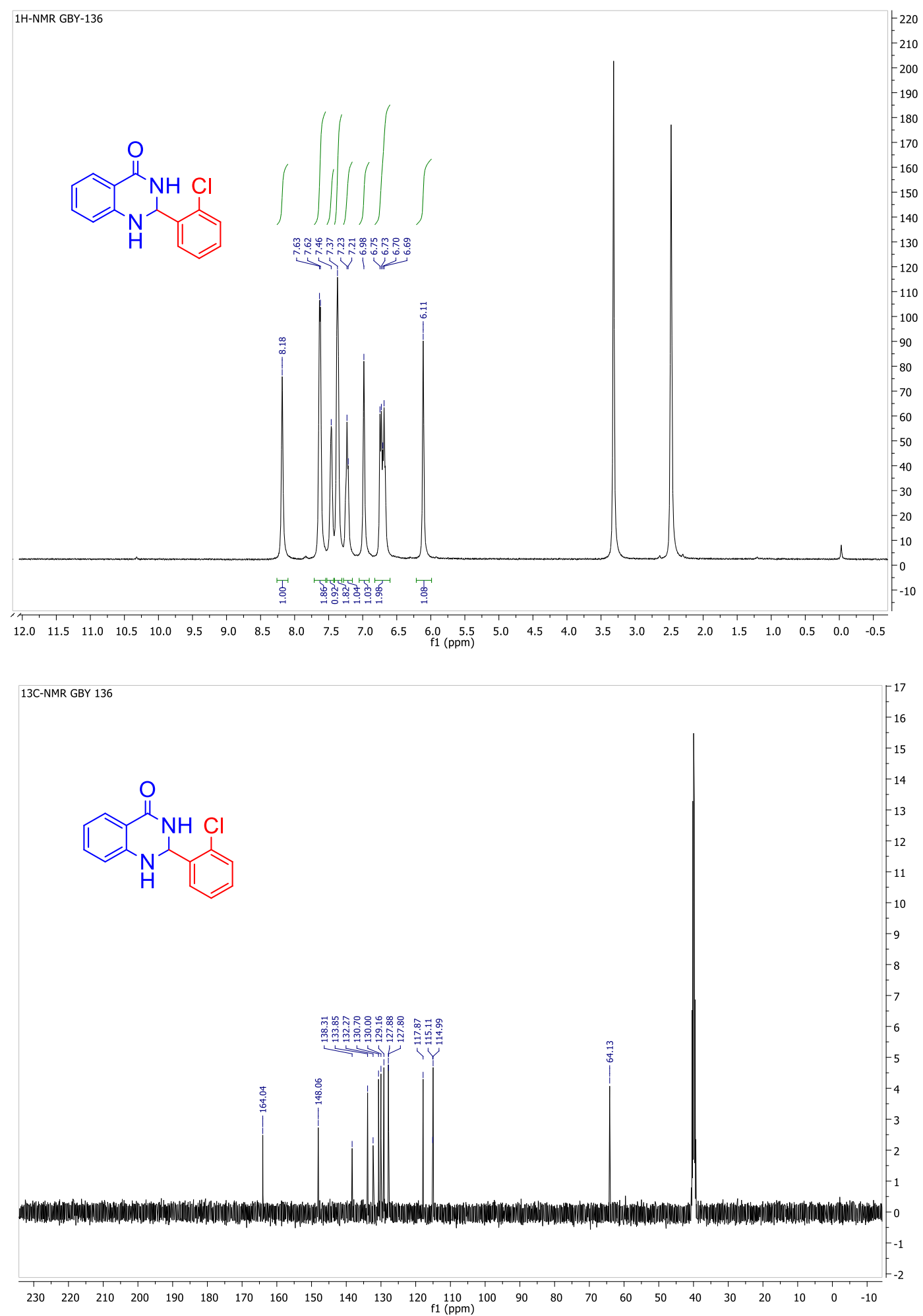

Figure S8. ${ }^{1} \mathrm{H}\left(400 \mathrm{MHz}, \mathrm{DMSO}-d_{6}\right)$ and ${ }^{13} \mathrm{C}$ NMR $\left(100 \mathrm{MHz}, \mathrm{DMSO}-d_{6}\right)$ spectral reproduction of 2(2-chlorophenyl)-2,3-dihydroquinazolin-4(1H)-one (3d). 

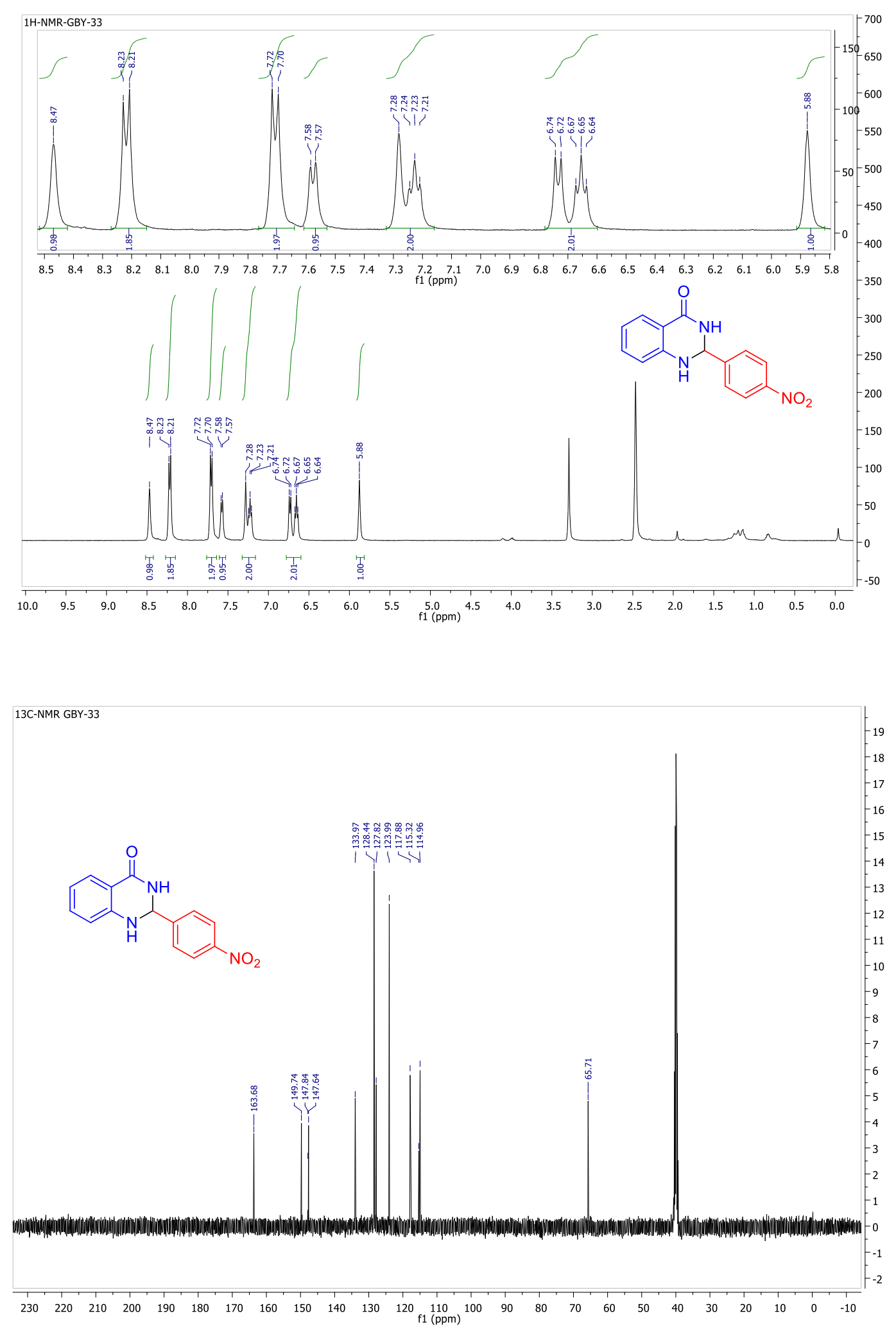

Figure S9. ${ }^{1} \mathrm{H}\left(400 \mathrm{MHz}, \mathrm{DMSO}-d_{6}\right)$ and ${ }^{13} \mathrm{C} \mathrm{NMR}\left(100 \mathrm{MHz}, \mathrm{DMSO}-d_{6}\right)$ spectral reproduction of 2(4-nitrophenyl)-2,3-dihydroquinazolin-4(1H)-one (3h). 

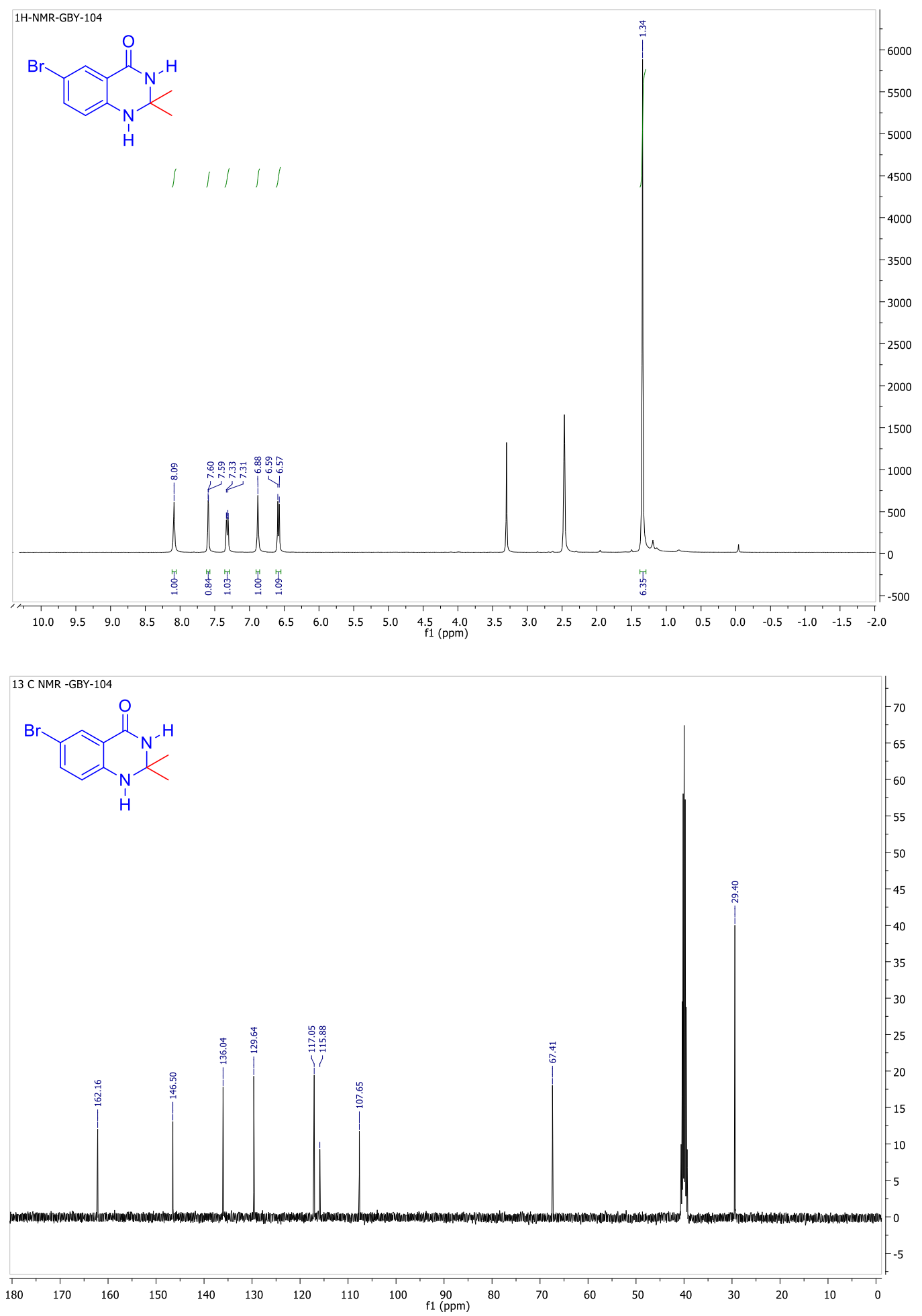

Figure S10. ${ }^{1} \mathrm{H}\left(400 \mathrm{MHz}, \mathrm{DMSO}-d_{6}\right)$ and ${ }^{13} \mathrm{C}$ NMR $\left(100 \mathrm{MHz}, \mathrm{DMSO}-d_{6}\right)$ spectral reproduction of 6-bromo-2,2-dimethyl-2,3-dihydroquinazolin-4(1H)-one $(\mathbf{8 b})$. 

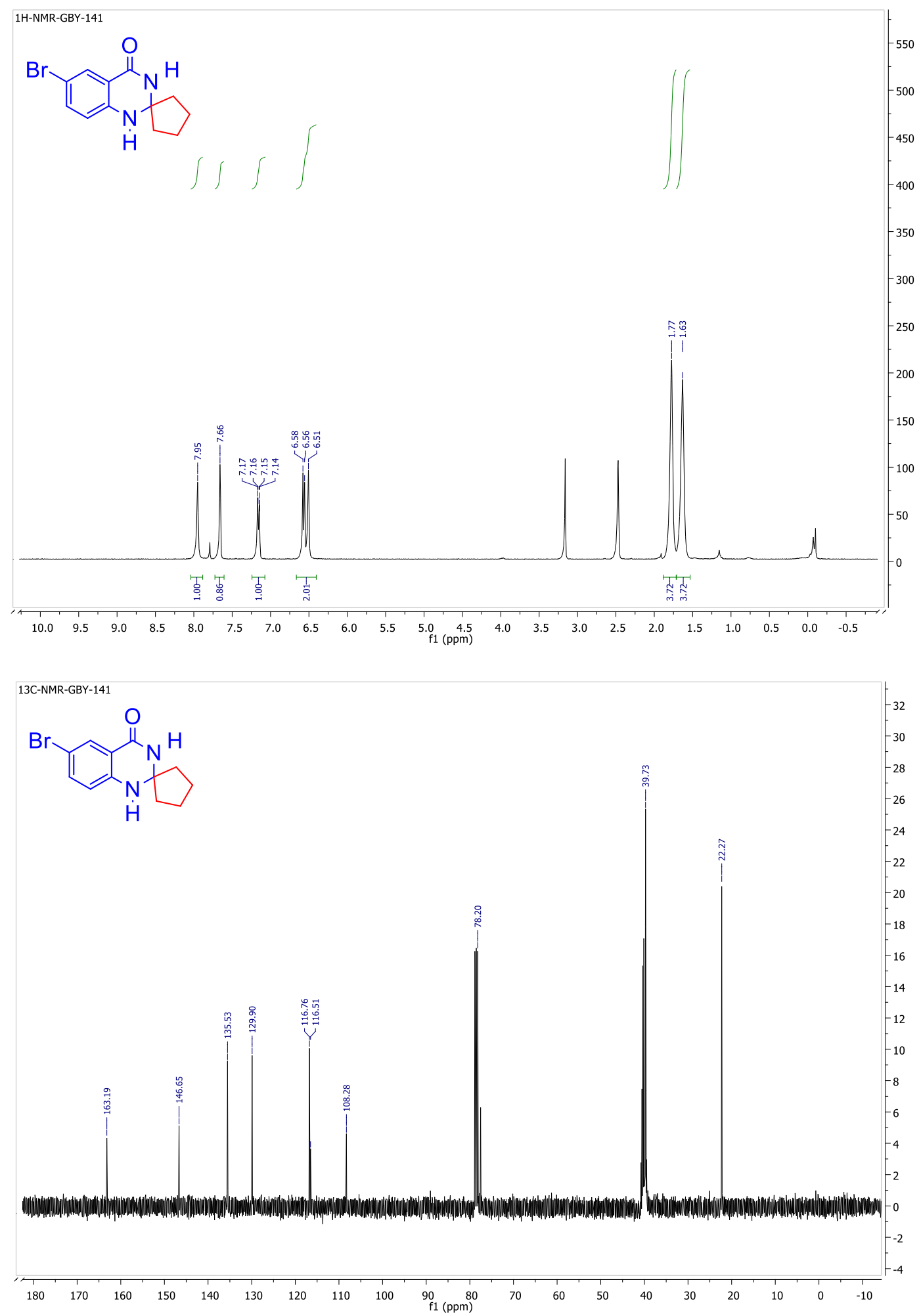

Figure S11. ${ }^{1} \mathrm{H}\left(400 \mathrm{MHz}, \mathrm{DMSO}-d_{6}\right)$ and ${ }^{13} \mathrm{C}$ NMR $\left(100 \mathrm{MHz}\right.$, DMSO- $\left.d_{6}\right)$ spectral reproduction of 6'-bromo-1'H-spiro[cyclopentane-1,2'-quinazolin]-4'(3'H)-one (9b). 

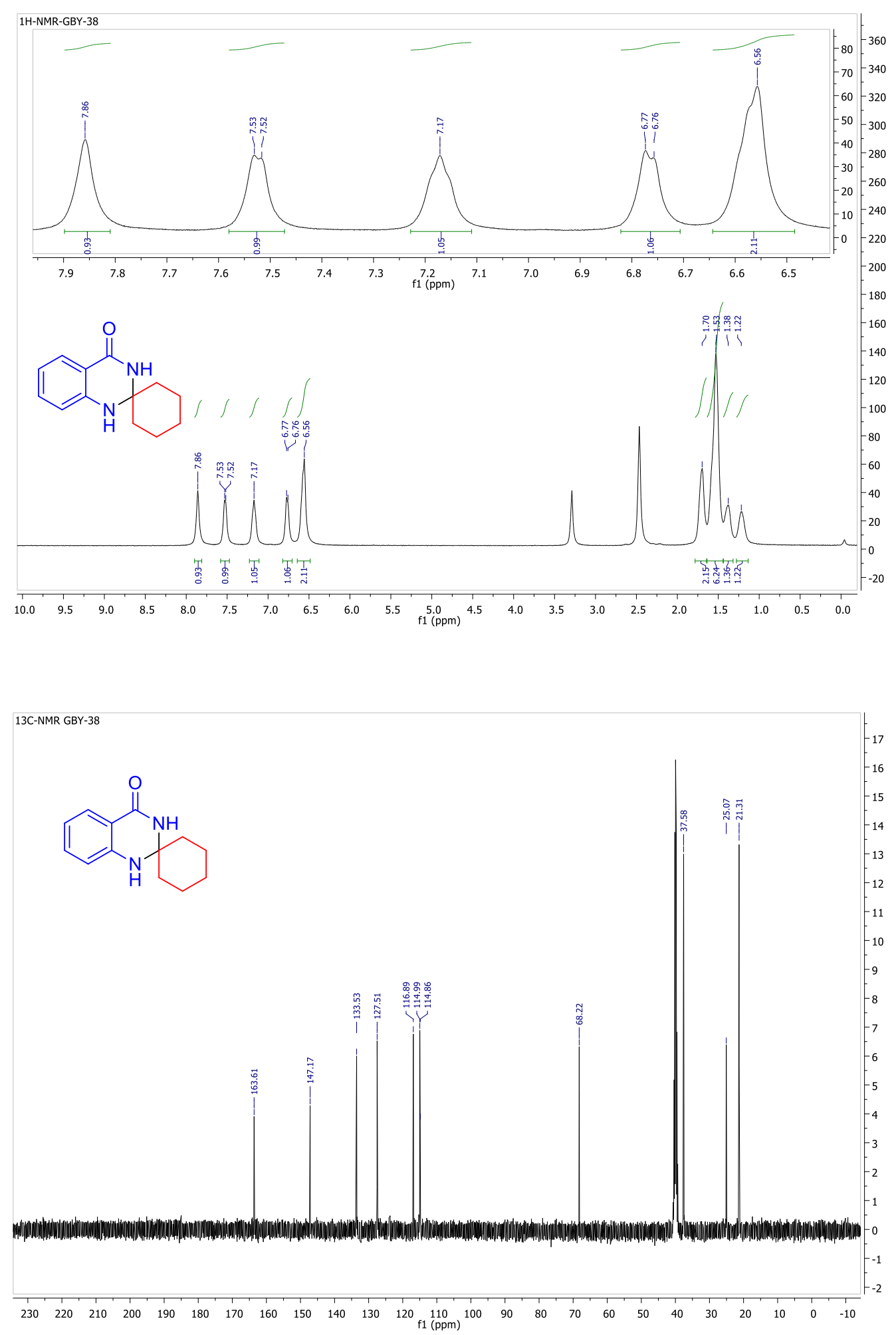

Figure S12. ${ }^{1} \mathrm{H}\left(400 \mathrm{MHz}, \mathrm{DMSO}-d_{6}\right)$ and ${ }^{13} \mathrm{C}$ NMR $\left(100 \mathrm{MHz}, \mathrm{DMSO}-d_{6}\right)$ spectral reproduction of 1'H-spiro[cyclohexane-1,2'-quinazolin]-4'(3'H)-one (10a). 

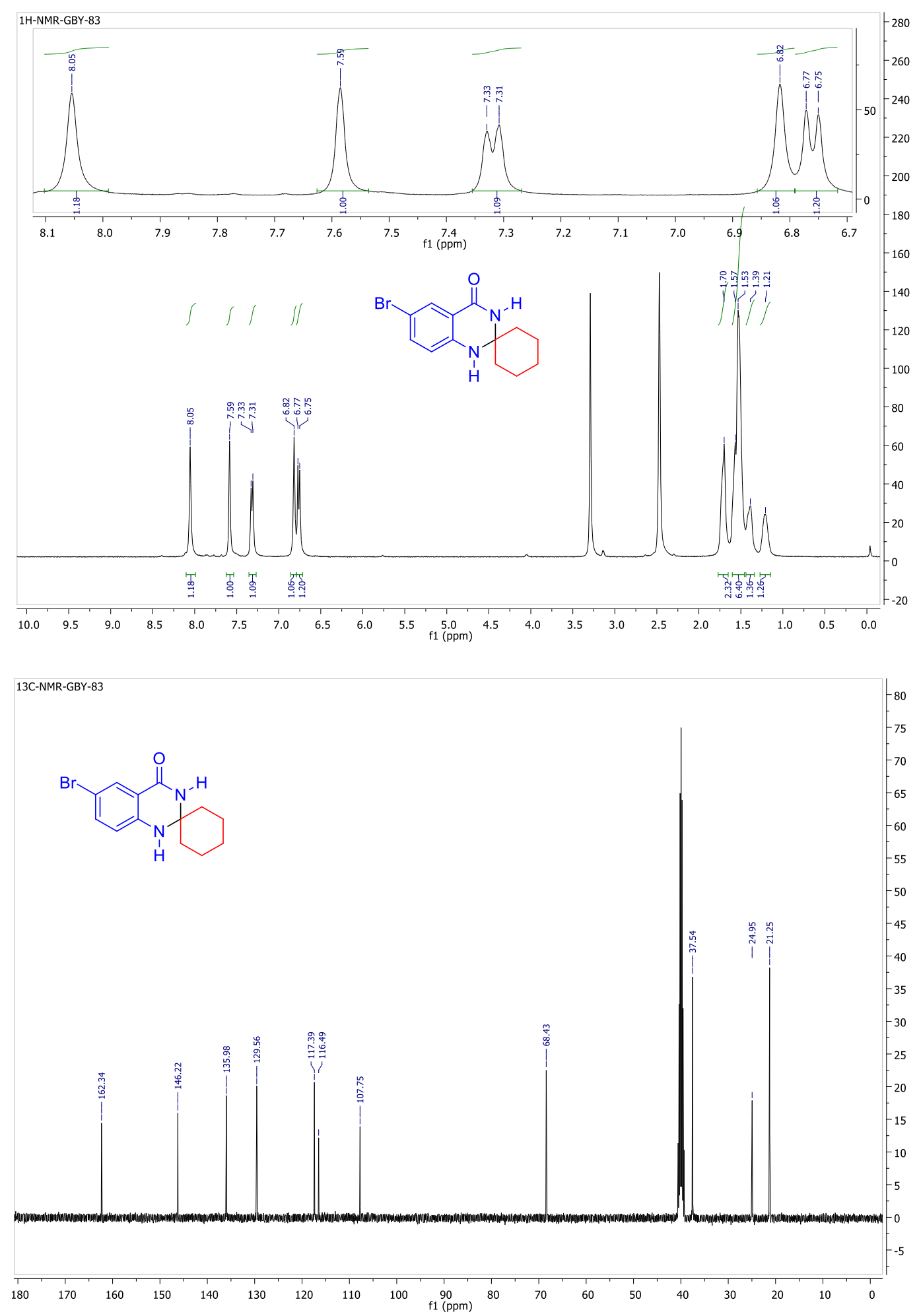

Figure S13. ${ }^{1} \mathrm{H}\left(400 \mathrm{MHz}, \mathrm{DMSO}-d_{6}\right)$ and ${ }^{13} \mathrm{C}$ NMR $\left(100 \mathrm{MHz}\right.$, DMSO- $\left.d_{6}\right)$ spectral reproduction of 6'-bromo-1'H-spiro[cyclohexane-1,2'-quinazolin]-4'(3'H)-one (10b). 

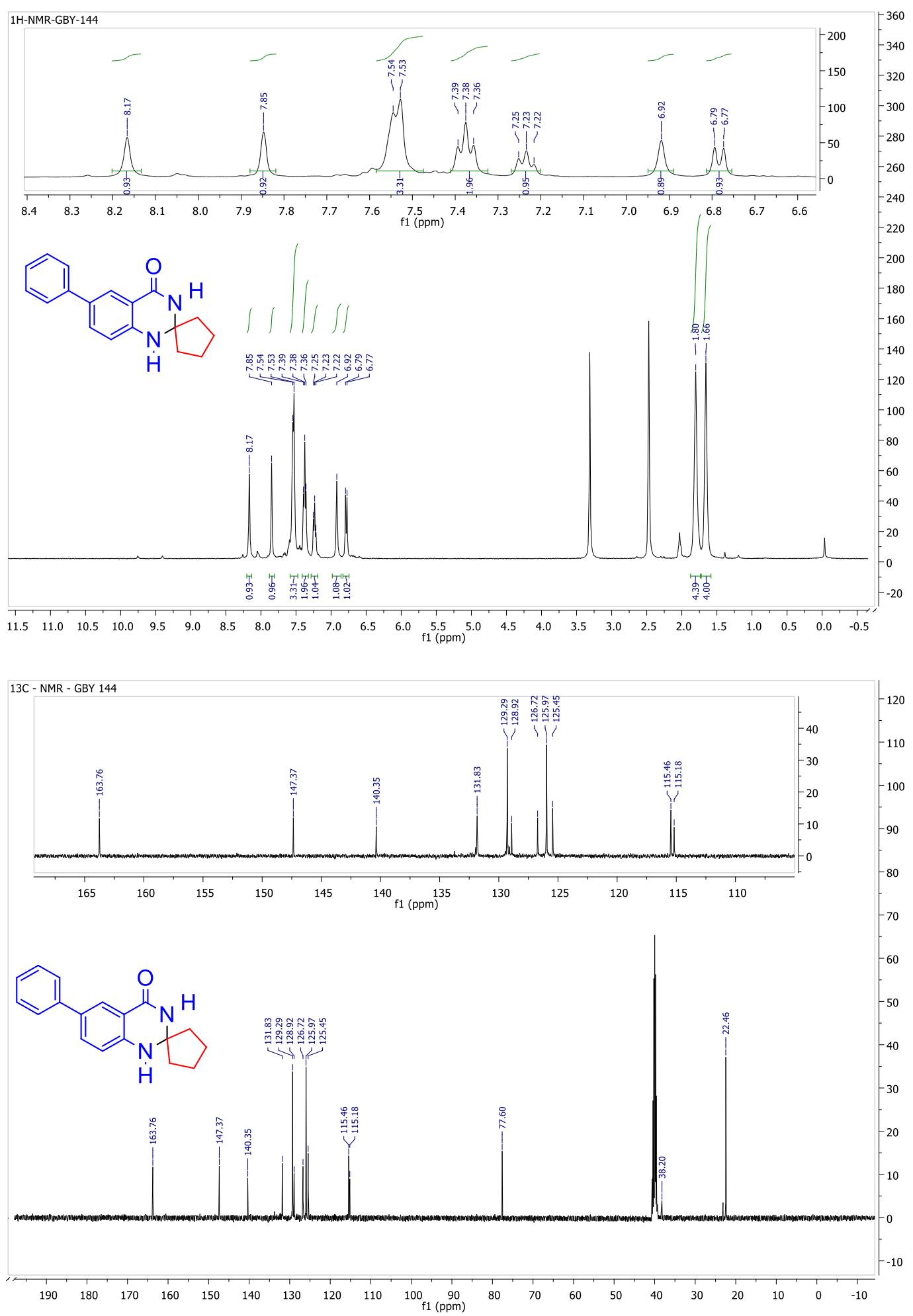

Figure S14. ${ }^{1} \mathrm{H}\left(400 \mathrm{MHz}, \mathrm{DMSO}-d_{6}\right)$ and ${ }^{13} \mathrm{C}$ NMR $\left(100 \mathrm{MHz}, \mathrm{DMSO}-d_{6}\right)$ spectral reproduction of 6'-phenyl-1'H-spiro[cyclopentane-1,2'-quinazolin]-4'(3'H)-one (10c). 

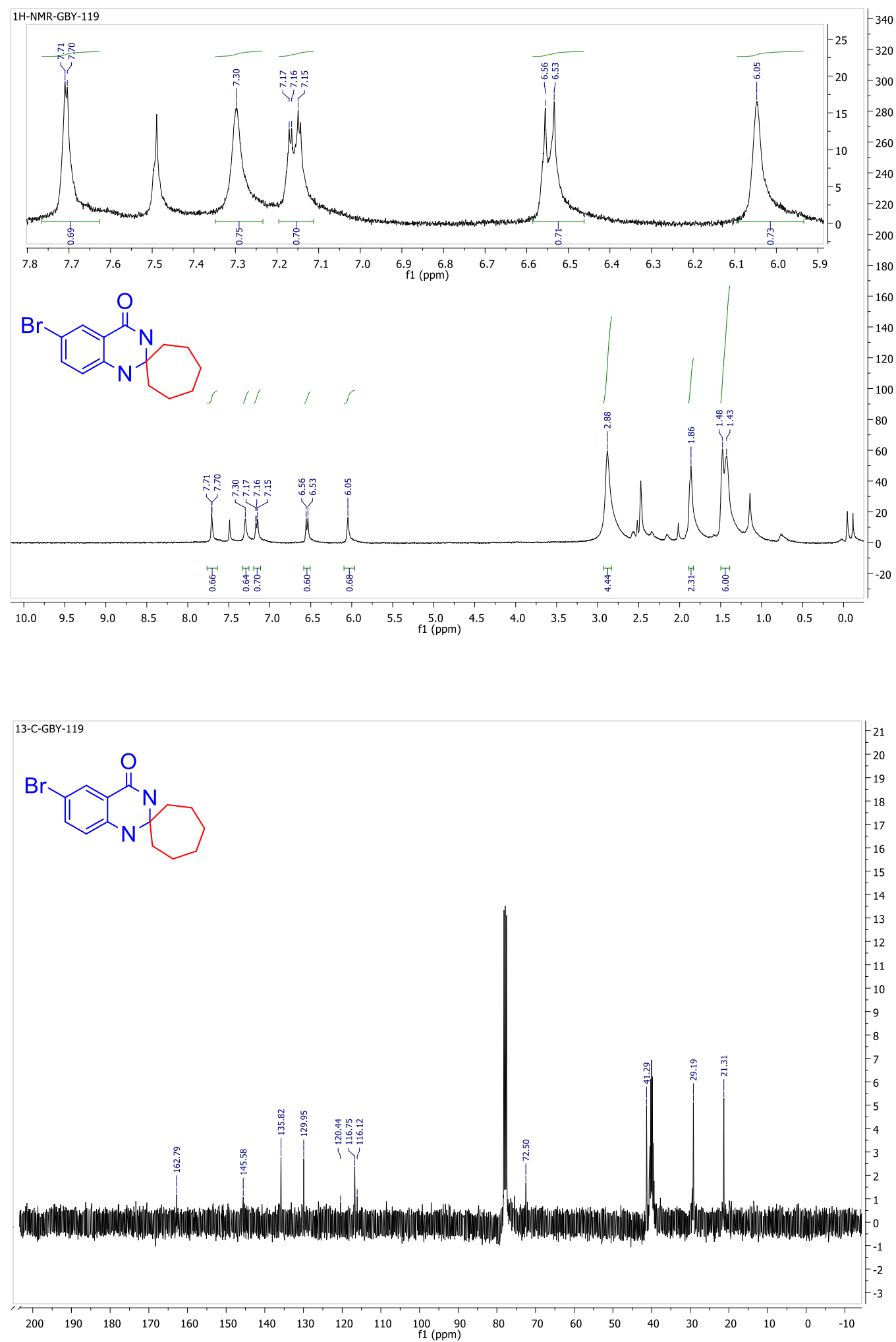

Figure S15. ${ }^{1} \mathrm{H}\left(400 \mathrm{MHz}, \mathrm{DMSO}-d_{6}\right)$ and ${ }^{13} \mathrm{C}$ NMR (100 MHz, DMSO- $\left.d_{6}\right)$ spectral reproduction of 6'-bromo-1'H-spiro[cycloheptane-1,2'-quinazolin]-4'(3'H)-one (11b). 

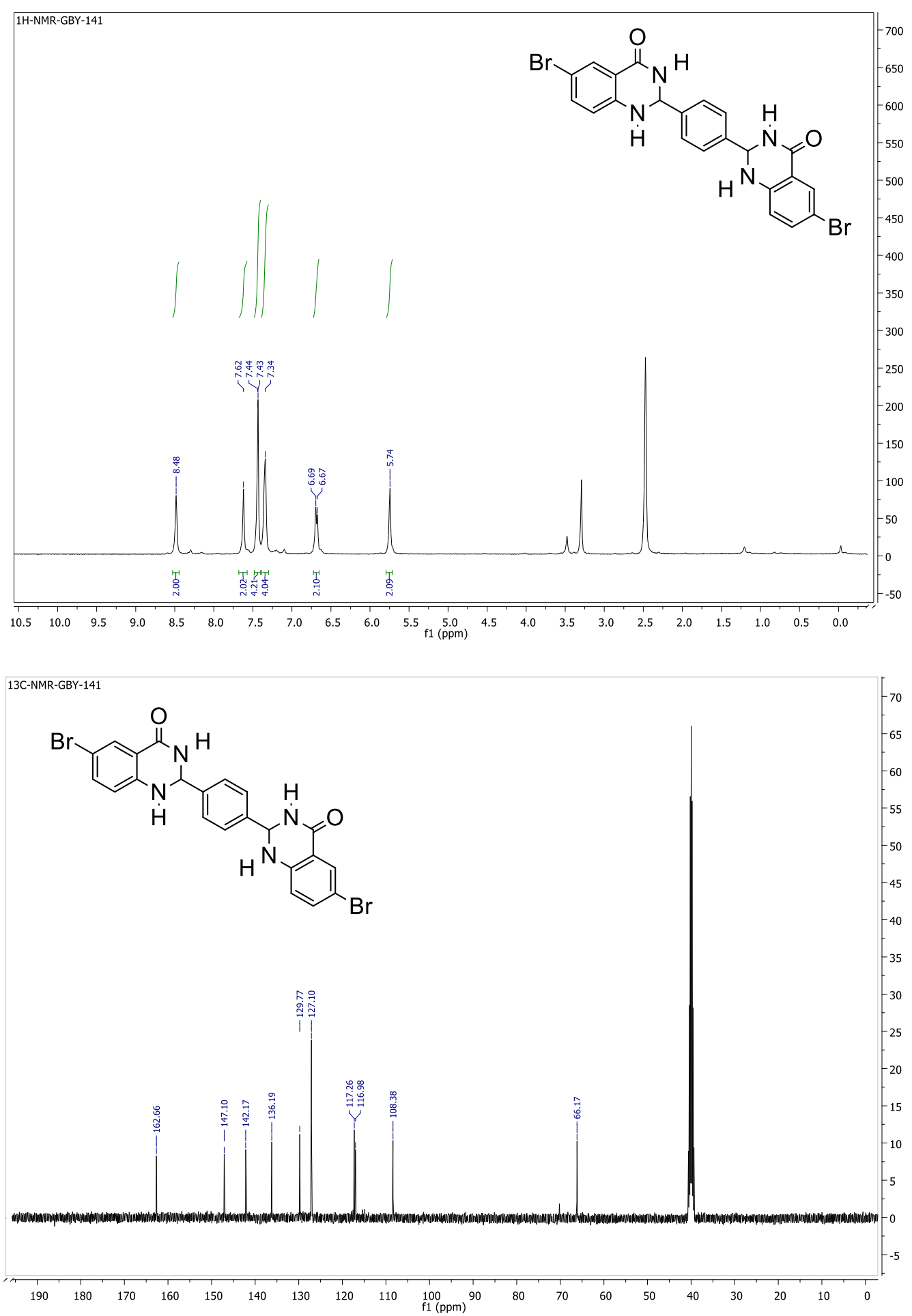

Figure S16. ${ }^{1} \mathrm{H}\left(400 \mathrm{MHz}, \mathrm{DMSO}-d_{6}\right)$ and ${ }^{13} \mathrm{C}$ NMR $\left(100 \mathrm{MHz}\right.$, DMSO- $\left.d_{6}\right)$ spectral reproduction of 2,2'-(1,4-phenylene)bis(6-bromo-2,3 dihydroquinazolin-4(1H)-one (13b). 


\section{References}

1. A. A. Khan, K. Mitra, A. Mandal, N. Baildya, M. A. Mondal, Heteroatom Chem. 2017, 28, 19.

2. H. R. Safaei, M. Shekouhy, S. Ghorbanzadeh, ChemistrySelect, 2018, 3, 4750-4759, DOI.org/10.1002/slct.201800456.

3. D. Rambabu, S. K. Kumar, B. Y. Sreenivas, S. Sandra, A. Kandale, P. Misra, M. V. Rao, M. Pal, Tetrahedron Lett. 2013, 54, 495-501, DOI.org/10.1016/j.tetlet.2012.11.057.

4. S. Das, S. Santra, S. Jana, G.Zyryanov, A.Majee, A. Hajra, Eur. J. Org. Chem. 2017, 33 ,49554962, DOI.org/10.1002/ejoc.201700966.

5. M. Hajjami, A. Choghamarani, R. Nejad, B. Tahmasbi, New J. Chem. 2016, 40, 3066-3074, DOI.10.1039/C5NJ03546E.

6. A. Gharib, B. Khorasani, M. Jahangir, M. Roshani, R. Safaee, Org. Chem. Int. 2013, 848237, $1-14$.

7. H. Alinezhad, E. Soleymani, M. Zare , Research on Chemical Intermediates, 2016, 43, 457466.

8. B. Dam, R. Patil, Yuan-Ron-Ma, A. Pal, New J. Chem. 2017, 41, 6553-6563, DOI.10.1039/C7NJ01208J.

9. S. Ayyanar, P. Vijaya, M. Mariyappan, V. Ashokkumar, V. Sadhasivam, S. Balkrishnan, C. Chinnadurai, S. Murgesan, New J. Chem. 2017, 41, 7980-7986, DOI.10.1039/C7NJ00538E.

10. M. Sharma, S. Pandey, K. Chauhan, D. Sharma, B. Kumar, P. Chauhan, J. Org. Chem. 2012, 77, 929-937, DOI.org/10.1021/jo2020856.

11. M. Beyki, F. Mehrjardi, Lett. Org. Chem. 2017, 15, 39-44, DOI: $10.2174 / 1570178614666170711144740$.

12. K.V.Aken, L. Strekowaski, L.Patiny, Beilstein J. Org. Chem. 2006, 2, No3, DOI:10.1186/18605397-2-3

13. H. Kiyani, M. Tazari, F. Ghorbani, Lett.Org. Chem., 2018, 15, 523-529, DOI.org/10.2174/1570178614666170710094547.

14. M. Rahman, I. Ling, N. Abdullah, R. Hashim, A. Hajra, $R S C A d v$., 2015, 5, 7755-7760, DOI.10.1039/C4RA16374E.

15. F. Tamaddon, M.T.K. Varnamkhasti Current Catalysis, 2017, 6, 57-66, DOI: $10.2174 / 2211544705666161018155755$.

16. Y. Luo, Y. Wu, Y. Wang, H. Sun, Z. Xie, W. Zhang, Z. Gao, RSC Adv., 2016, 6, 66074-66077, DOI.10.1039/C6RA14583C.

17. M. Sharma, S. Pandey, K. Chauhan, D. Sharma, B. Kumar, P. Chauhan, J. Org. Chem., 2012, 77, 929-937, DOI.org/10.1021/jo2020856.

18. B. Majumdar, S. Mandani, T. Bhattacharya, D. Sarma, T. Sarma, J. Org. Chem., 2017, 82, 2097-2106, DOI.org/10.1021/acs.joc.6b02914.

19. W. Zhao, W. Ma, T. Xiao, F. Li, ChemistrySelect, 2017, 2, 3608-3612, DOI.org/10.1002/slct.201700780.

20. S. K. Ghosh, R. Nagarajan, RSC Adv., 2016, 6, 27378-27387, DOI.10.1039/C6RA00855K. 